\title{
Demonstration with Special TCI-15 Datasets of Potential Impacts of New-Generation Satellite Atmospheric Motion Vectors on Navy Regional and Global Models
}

\author{
Russell L. Elsberry, ${ }^{\mathrm{a}}$ Eric A. Hendricks, ${ }^{\mathrm{b}}$ Christopher S. Velden, ${ }^{\mathrm{c}}$ Michael M. Bell, ${ }^{\mathrm{d}}$ \\ MELINDA PenG, ${ }^{\mathrm{e}}$ Eleanor CASAS, ${ }^{\mathrm{d}}$ AND QINGYUn ZHAO ${ }^{\mathrm{f}}$ \\ ${ }^{\text {a }}$ University of Colorado Colorado Springs, Colorado Springs, Colorado \\ ${ }^{\mathrm{b}}$ Naval Postgraduate School, Monterey, California \\ ${ }^{\mathrm{c}}$ Cooperative Institute Meteorological Satellite Studies, Madison, Wisconsin \\ ${ }^{\mathrm{d}}$ Colorado State University, Fort Collins, Colorado \\ ${ }^{\mathrm{e}}$ CSRA, and Naval Research Laboratory, Monterey, California \\ ${ }^{\mathrm{f}}$ Naval Research Laboratory, Monterey, California
}

(Manuscript received 16 November 2017, in final form 2 October 2018)

\begin{abstract}
A dynamic initialization assimilation scheme is demonstrated utilizing rapid-scan atmospheric motion vectors (AMVs) at 15-min intervals to simulate the real-time capability that now exists from the new generation of geostationary meteorological satellites. The impacts of these AMVs are validated with special Tropical Cyclone Intensity Experiment (TCI-15) datasets during 1200-1800 UTC 4 October leading up to a NASA WB-57 eyewall crossing of Hurricane Joaquin. Incorporating the AMV fields in the Spline Analysis at Mesoscale Utilizing Radar and Aircraft Instrumentation (SAMURAI) COAMPS Dynamic Initialization (SCDI) means there are 30 and 90 time steps on the 15- and 5-km grids, respectively, during which the mass fields are adjusted to these AMV-based wind increments during each 15-min assimilation period. The SCDI analysis of the three-dimensional vortex structure of Joaquin at 1800 UTC 4 October closely replicates the vortex tilt analyzed from the High-Definition Sounding System (HDSS) dropwindsondes. Vertical wind shears based on the AMVs at 15-min intervals are well correlated with the extreme rapid decay, an interruption of that rapid decay, and the subsequent period of constant intensity of Joaquin. Utilizing the SCDI analysis as the initial conditions for two versions of the COAMPS-TC model results in an accurate 72-h prediction of the interruption of the rapid decay and the period of constant intensity. Upscaling a similar SCDI analysis based on the 15-min interval AMVs provides a more realistic intensity and structure of Tropical Storm Joaquin for the initial conditions of the Navy Global Environmental Model (NAVGEM) than the synthetic TC vortex used operationally. This demonstration for a single 6-h period of AMVs indicates the potential for substantial impacts when an end-to-end cycling version is developed.
\end{abstract}

\section{Introduction}

The objective of this study is to demonstrate the potential impacts for the U.S. Navy regional and global models of continuous rapid-scan atmospheric motion vectors at 10 -min intervals that simulate the real-time capability that now exists over the full disk of the new generation of geostationary meteorological satellites. The Advanced Himawari Imager (AHI) and the GOES-16 with the Advanced Baseline Imager (Schmit et al. 2017) have 16 spectral bands that can simultaneously scan a full Earth disk at 10-min intervals. The spatial resolution is also enhanced to $0.5 \mathrm{~km}$ for band 3 in the visible wavelength, and the two other

Corresponding author: Russell L. Elsberry, relsberr@uccs.edu visible bands and band 4 in the near-infrared have $1-\mathrm{km}$ resolution. Two other near-infrared bands and all infrared bands have a spatial resolution of $2 \mathrm{~km}$. The infrared band 11 is used for detection of thin ice cloud such as is often found at the edge of the TC cirrus outflow, and infrared band 16 is used for cloud-top height assignment. The three water vapor bands are most sensitive to the middle- to upper-tropospheric humidity.

Given this high spatial, spectral, and temporal resolution, these new-generation imagers are better able to track coherent clouds and water vapor features to derive atmospheric motion vectors (AMVs) that provide estimates of tropospheric winds (Velden et al. 2005). That is, clouds or water vapor features can be selected from an image at time $t$ and then the backward and forward motion vectors from $t-10$ min to time $t$ and from time 
$t$ to $t+10 \mathrm{~min}$ can be averaged to calculate AMVs at time $t$ (rather than $\pm 30 \mathrm{~min}$ as in the past). The individual AMVs are assigned heights (pressure levels) near the cloud top or for water vapor features using multispectral techniques with accuracies of $\pm 25 \mathrm{hPa}$. Some limitations of present-day AMVs are that 30-min sampling frequency for tracking clouds is not optimal (Velden et al. 2005), and more rapid scanning sequences (1-5-min images) have been limited to restricted domains and durations. More importantly, applications in numerical weather prediction (NWP) have often been constrained to 6-h data assimilation cycles and AMV dataset thinning. In this study it will be demonstrated that shorter sampling times and continuous rapid scanning combined with the advanced sensors on these newgeneration satellites will substantially improve the quality and quantity of the AMVs, and thus their potential impacts on the U.S. Navy regional and global model analyses and predictions.

Oyama (2017) has demonstrated that composites of TC outflows at the cloud tops computed from AMVs can be related to the intensification rates. Oyama et al. (2018) then compare the maximum tangential winds in the outflow estimated from 30-min-interval Himawari-8 target observations with the maximum surface wind evolutions throughout the lifetime of two typhoons and suggest that Himawari-8 AMVs can be used to monitor TC intensification and related structural changes.

In this study, a special set of GOES-East rapid-scan AMVs will provide a near-continuous record every $15 \mathrm{~min}$ of the horizontal mass flux at the top of an evolving TC outflow layer. The development or inhibition of channeled outflow patterns, which are indicative of inertial stability or ventilation conditions that modulate the TC intensity, will be better captured. In addition, the ability to track smaller lowertropospheric cumulus clouds in the high-resolution visible and near-infrared channels will provide abundant AMVs outside the cirrus cloud shield to analyze the outer TC vortex wind structure as well as represent the environmental mass influx. In conjunction with AMVs derived from the water vapor channels, the middle-tropospheric moisture flux from the environment to the outer regions of the TC can be continuously monitored.

Although the details will not be provided in this model proof-of-concept demonstration, it may be useful to at least describe the expected model physical linkages connecting the improved AMV depiction outflow with the intensity changes. First, where AMVs are outward directed relative to the convective cloud bands and cirrus cloud edges, they are expected to indicate divergence and convergence associated with ascent in deep convection and adjacent subsidence-induced clearing. In response, the model physics will infer the vertical ascent/ descent and thus the convective heating distribution in the horizontal and vertical, which will lead to modification of the pressure (mass) field. Model dynamics will then adjust the mean and asymmetric wind fields, which in the lower model levels will take into account the planetary boundary layer frictional effects and enthalpy fluxes. Whereas these internal adjustments will determine the intensity change, the TC vortex dynamics and physics prediction are expected to also improve the interaction between the vortex and its environment in conjunction with the better depiction of the outflow jets from the high temporal and spatial resolution AMVs.

In section 2, the special AMV and other datasets obtained during the Tropical Cyclone Intensity Experiment (TCI-15; Doyle et al. 2017) will be used to demonstrate how the nearly continuous AMVs from the new-generation satellites might be utilized to improve TC analyses and predictions in the U.S. Navy regional and global models. The focus of this demonstration is on a 6-h dataset of 15-min-interval AMVs that was reprocessed by Cooperative Institute for Meteorological Satellite Studies (CIMSS) from GOES-East rapid-scan imagery during the interruption of a rapid decay period during Hurricane Joaquin (2015). The U.S. Navy regional and global models used in these tests are described in section $2 \mathrm{~b}$, and the analysis technique used to spread the AMV information to the grid points of the U.S. Navy regional model is described in section 2c. A dynamic initialization technique has been developed in which AMV-based wind increments relative to the regional numerical model background wind forecast are utilized at 15 -min intervals for the 6 -h period when special TCI-15 in situ datasets were available for validation (section 2d). It will be demonstrated in section $2 \mathrm{e}$ that the vortex structure in this dynamic initialization closely resembles the observed vortex tilt analyzed from a set of High-Definition Sounding System (HDSS) soundings deployed at a spacing of $4.5 \mathrm{~km}$ during an aircraft center overpass of Joaquin.

This dynamic initialization analysis is then utilized in section 3 as the initial conditions for the U.S. Navy regional model forecast for comparison with the real-time model forecast that utilizes a bogus TC vortex. Similarly, it will be demonstrated in section 4 that the regional analysis of the TC intensity and structure can be upscaled to the U.S. Navy global model grid to provide more realistic initial conditions than a synthetic TC vortex. While the objective here is only to demonstrate the concept with a single case study, future studies will utilize all of the forty 6-h AMV datasets to optimize the analysis technique that spreads the AMV information, 
and more fully demonstrates the impacts of the AMVs on the regional and global model forecasts. An important goal of these future studies will be to demonstrate that a global model forecast after $6 \mathrm{~h}$ can then provide the initial and lateral boundary conditions for the next dynamic initialization, and this cycling can be an end-toend NWP application that fully utilizes the information content of the high temporal and spatial resolution AMV datasets.

\section{Methodology}

\section{a. Special reprocessed AMV datasets}

Velden et al. (2017) provide an excellent review of the recent advances in the calculation of AMVs and their utilization to improve TC forecasts in NWP. In their study, enhanced AMV observations at hourly intervals were produced by utilizing special rapid-scan periods from the GOES-East and Meteosat satellites and adjusting the target selection and search box criteria to allow a more dense level of coverage of AMVs over three Atlantic hurricanes and their near environments. With this special processing strategy, these AMV observations in effect serve as a proxy for the AMV datasets becoming routinely available from the Himawari-8 and GOES-16 satellites (except for the limited coverage of the rapidscan domains).

Velden et al. (2017) utilized the operational version of the Hurricane Weather and Research Forecast (HWRF) Model data assimilation to create "cold start" initial conditions for the HWRF forecast model throughout the life cycles of the three hurricanes. A special feature of the blending initialization in this version of the HWRF eliminated analysis increments within $150 \mathrm{~km}$ of the TC center and below $600 \mathrm{mb}(1 \mathrm{mb}=1 \mathrm{hPa})$ in order to maintain an enhanced vortex structure based on the initial TC intensity and wind radii provided by the National Hurricane Center (NHC). Despite some relatively small intensity forecast errors for both $\mathrm{Su}$ perstorm Sandy (2012) and Hurricane Edouard (2014) by the operational HWRF, modest forecast improvements were achieved from the AMV-enhanced initial conditions. The early rapid intensification stage of Hurricane Gonzalo (2014) was not well forecast by the operational HWRF model, and the AMVenhanced initial conditions did not result in any intensity forecast improvements, which may be attributed to the limited usage of AMVs in the inner region of the TC. In addition, only a 6-h assimilation cycle was possible.

Kim et al. (2017) have also examined the observation impact of enhanced AMV datasets created by CIMSS for two typhoons in the western North Pacific during
September 2008. These AMV datasets also utilized rapidscan images (when available) versus the normal 30-min images from the Multifunctional Transport Satellites (MTSAT). As in Velden et al. (2017), the targeting routines were set to achieve a higher AMV density and the postprocessing analysis and quality control processes were adjusted to better represent smaller-scale flow fields. Kim et al. used an adjoint-based observation impact method and observing system experiments to examine the impact of these enhanced AMVs in model TC forecasts, and they documented improved track forecasts. Furthermore, Kim et al. demonstrated that continuous cycling of the enhanced hourly AMVs improved both the model background and the initial analyses.

In this study, enhanced AMV datasets were created by CIMSS each $15 \mathrm{~min}$ beginning at 1215 UTC 26 September 2015 (prior to the start of the best track) of Hurricane Joaquin and continuing to 2345 UTC 6 October. Specifically, the focus will be on the impact of the AMVs during 1200-1800 UTC 4 October when Hurricane Joaquin was moving northeastward and was rapidly decaying (Berg 2016; Creasey and Elsberry 2017). An example of the enhanced AMV coverage at 1245 UTC 4 October is given in Fig. 1, in which primarily over-ocean AMVs are plotted. While the colors associated with these AMVs indicate the pressure heights within various binned ranges (legend in top-left corner), it is important to note that each AMV is assigned a discrete pressure height to the nearest $1 \mathrm{hPa}$. Quality indices are provided for the AMVs based on quality control tests in a postprocessing step (Velden et al. 2017). The AMVs are calculated from GOESEast channels in the visible, infrared, and water vapor bands. Over the domain selected for this study (roughly $1^{\circ}-44^{\circ} \mathrm{N}, 30^{\circ}-86^{\circ} \mathrm{W}$ ), a typical dataset would include 15000-20000 AMVs.

The objective in this study is to utilize as many of these 15-min AMVs as possible without the thinning (or superob approach) that is typically utilized for operational models. Velden et al. (2017) utilized a stringent quality control process that slightly decreased the number of AMVs in the region of the TCs (see their Fig. 1), but their HWRF data assimilation did not utilize any AMVs between 400 and $700 \mathrm{hPa}$.

\section{b. Numerical models employed}

The Coupled Ocean-Atmosphere Mesoscale Prediction System for Tropical Cyclones (COAMPS-TC; Doyle et al. 2014) is an operational regional numerical model for TC prediction that is triple nested $(45,15$, and $5 \mathrm{~km}$ ) and thus has the horizontal resolution to resolve the enhanced AMV datasets. However, the previous data assimilation system for COAMPS-TC caused such 


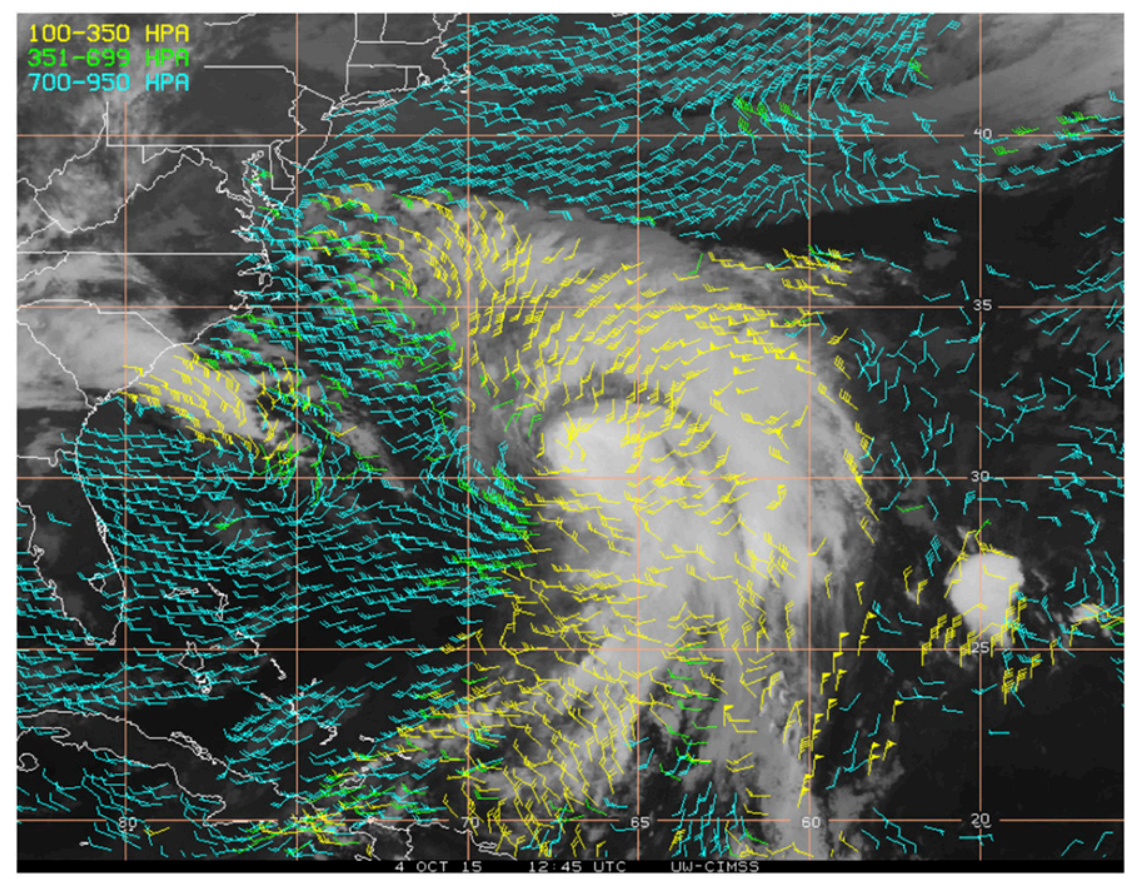

FIG. 1. Reprocessed AMVs surrounding Hurricane Joaquin at 1245 UTC 4 Oct 2015 provided by the CIMSS AMV team. The plot is color coded to depict pressure-elevation ranges (hPa) within which the individual AMV height assignments are found.

a large spindown in the first $6-12 \mathrm{~h}$ that the intensity forecast was adversely affected throughout the forecast interval (Hendricks et al. 2011). Thus, the COAMPS-TC presently is cold started with interpolated initial fields from the U.S. Navy and the National Centers for Environmental Prediction (NCEP) global model data assimilation systems that are quasi-balanced in terms of the mass and wind fields as the initial time. Another issue is that the upper-tropospheric AMVs in TCs are expected to have large magnitudes in the outflow channel and vary rapidly on small scales near the center. Inserting these upper-tropospheric AMVs without supporting mass fields into the initial conditions derived from a 6-h integration of the Navy Global Environmental Model (NAVGEM; Hogan et al. 2014) would likely exacerbate imbalances in the COAMPS-TC model. Although a four-dimensional variational data assimilation (4DVAR) for COAMPS-TC is under development, it is not yet available.

It is therefore concluded that the full information content of the Himawari-8 or GOES-16 AMV observations will not be realized until the data assimilation/ modeling system is redesigned to utilize these high temporal and spatial resolution wind datasets. Although a global 4DVAR system such as the Navy Data Assimilation System (NAVDAS-AR; Xu et al. 2005) has a better capability to handle more continuous AMV observations, the present plan at Naval Research Laboratory-Monterey is only to utilize hourly AMVs. Moreover, the NAVDASAR system has a spectral resolution that is more coarse than the NAVGEM model, so it will not be compatible with the high spatial resolution of the Himawari-8 or GOES-16 AMV observations. Consequently, an alternate dynamical initialization technique will be proposed in section $2 \mathrm{~d}$ that will utilize the full spatial and temporal resolution AMVs from the Himawari-8 or GOES-16 satellites.

\section{c. SAMURAI analysis methodology}

As indicated in section 2a above, the special AMV dataset is at 15-min intervals with latitude, longitude, and pressures that do not correspond to the horizontal and vertical grid points in the COAMPS-TC model. Therefore, the Spline Analysis at Mesoscale Utilizing Radar and Aircraft Instrumentation (SAMURAI; Bell et al. 2012) has been adapted to first spread the AMV speed and direction information horizontally and vertically to those COAMPS-TC grid points. More detailed descriptions of the SAMURAI analysis technique are provided in Bell et al. (2012) and Foerster and Bell (2017), and only a brief description is given here. The SAMURAI is a $3 D V A R$ analysis that minimizes an incremental form of a cost function using a finite-element approach with cubic spline elements as a basis. Observational error 
characteristics can be individually specified for different instrumentation options, but only the special satellite AMVs are used in this study. SAMURAI has three spatial filters that act as a background error covariance to spread information from the AMV observations throughout the domain: a Fourier filter, a third derivative constraint on the finite elements (Ooyama 2002), and a Gaussian recursive filter (Purser et al. 2003). For the current application, a combination of the spline-cutoff filter and the Gaussian recursive filter was used, with the filter wavelengths set to 10 nodal points and 6 nodal points, respectively.

In this application designed to simulate the impact of Himawari-8 or GOES-16 AMVs, SAMURAI produces AMV-based zonal and meridional wind increments every $15 \mathrm{~min}$ relative to a background COAMPS-TC 6-h forecast wind field. The 3DVAR solution with the spatial filter then provides the 3D wind vector increments $\left(\mathbf{V}_{s}-\mathbf{V}_{b}\right)$ at the COAMPS-TC horizontal grid points and pressure heights, where $\mathbf{V}_{s}$ is the SAMURAI-analyzed wind vector and $\mathbf{V}_{b}$ is the COAMPS-TC wind forecast vector during each 15 -min period for which an AMV dataset is available. These AMV-based wind increment fields are analyzed on each of the three COAMPS-TC nested grids (domain 1, $45 \mathrm{~km}$; domain $2,15 \mathrm{~km}$; and domain $3,5 \mathrm{~km}$ ) so that more and more of the spatial resolution of the AMVs near the TC center will be incorporated into domains 2 and 3 .

An example of the SAMURAI analysis wind increments relative to the background COAMPS-TC forecast wind field at 1630 UTC 4 October 2015 is given in Fig. 2. These wind increments at the $10.9-\mathrm{km}$ level over domain 2 indicate a large, coherent region about $1300-1800 \mathrm{~km}$ to the north of the center of Joaquin that has larger anticyclonic outflow with increment magnitudes as large as $5.5 \mathrm{~m} \mathrm{~s}^{-1}$. Furthermore, southwestward wind increments of $\sim 3.5 \mathrm{~m} \mathrm{~s}^{-1}$ to the west near $x=500 \mathrm{~km}$, $y=1600 \mathrm{~km}$ suggest that the AMVs have detected a stronger cyclonic trough than in the background COAMPS-TC forecast, which is then contributing to this larger outflow from Joaquin. To the east of Joaquin, a large region of northward AMV-based wind increments, again with magnitudes near $5.5 \mathrm{~m} \mathrm{~s}^{-1}$ relative to the COAMPS-TC 6 -h forecast wind field, indicates the equatorward branch of the outflow is too large in the COAMPS-TC forecast. The continuity of the wind increment analyses over such large regions suggests that the SAMURAI technique has spread the AMV information horizontally. Furthermore, the large gradient regions of these wind increments suggest areas of enhanced divergence or convergence relative to their distributions and magnitudes in the background

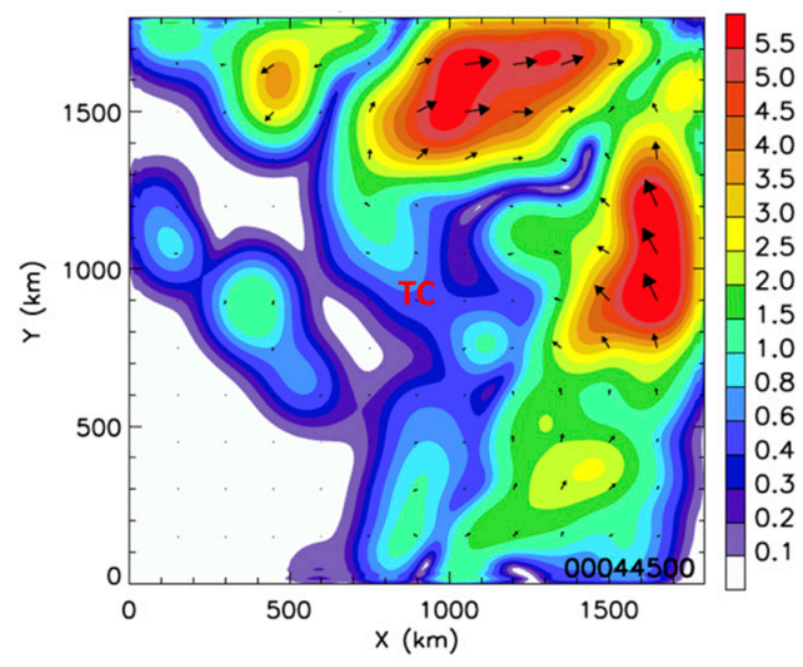

FIG. 2. Example of SAMURAI wind vector increments $\left(\mathrm{m} \mathrm{s}^{-1}\right)$ at $10.96 \mathrm{~km}$ over domain 2 relative to a background COAMPS-TC forecast wind field. Arrows indicate the wind increment direction, and the magnitudes are indicated by the color scale on the right.

COAMPS-TC forecast at that 15 -min period. The cumulative effect of these wind increments is calculated via the dynamic initialization technique described in the next subsection.

\section{d. Dynamic initialization methodology}

The SAMURAI/COAMPS-TC Dynamic Initialization (SCDI) is a simple nudging approach following Hendricks et al. (2011, 2013). In this application in which only AMVs are being incorporated, the nudging is for the zonal $u$ and meridional $v$ wind components as follows:

$$
\begin{aligned}
& \frac{\partial u}{\partial t}=F(u)+\alpha\left(u_{s}-u_{b}\right) \quad \text { and } \\
& \frac{\partial v}{\partial t}=F(v)+\alpha\left(v_{s}-v_{b}\right),
\end{aligned}
$$

where $F(u)$ and $F(v)$ are all other terms in the COAMPSTC momentum equations, $\alpha$ is the nudging coefficient $\left(0.0005 \mathrm{~s}^{-1}\right)$, and $\left(u_{s}-u_{b}, v_{s}-v_{b}\right)$ are the zonal and meridional components of the AMV-based SAMURAI vector wind increments described in section $2 b$.

A flowchart explaining the SCDI technique is presented in Fig. 3. At the first time in a series of 15-min AMVs, the COAMPS-TC initial conditions are a "cold start" from interpolated NAVGEM analyses at 0000 , 0600, 1200, or 1800 UTC to initialize all three grid domains. In the NAVGEM, a static balanced vortex matching the TC maximum wind is blended into the NAVDAS-AR global analysis near the warning position.

The first step in the SCDI (Fig. 3) is to integrate the background COAMPS-TC for $15 \mathrm{~min}$ until the time of 


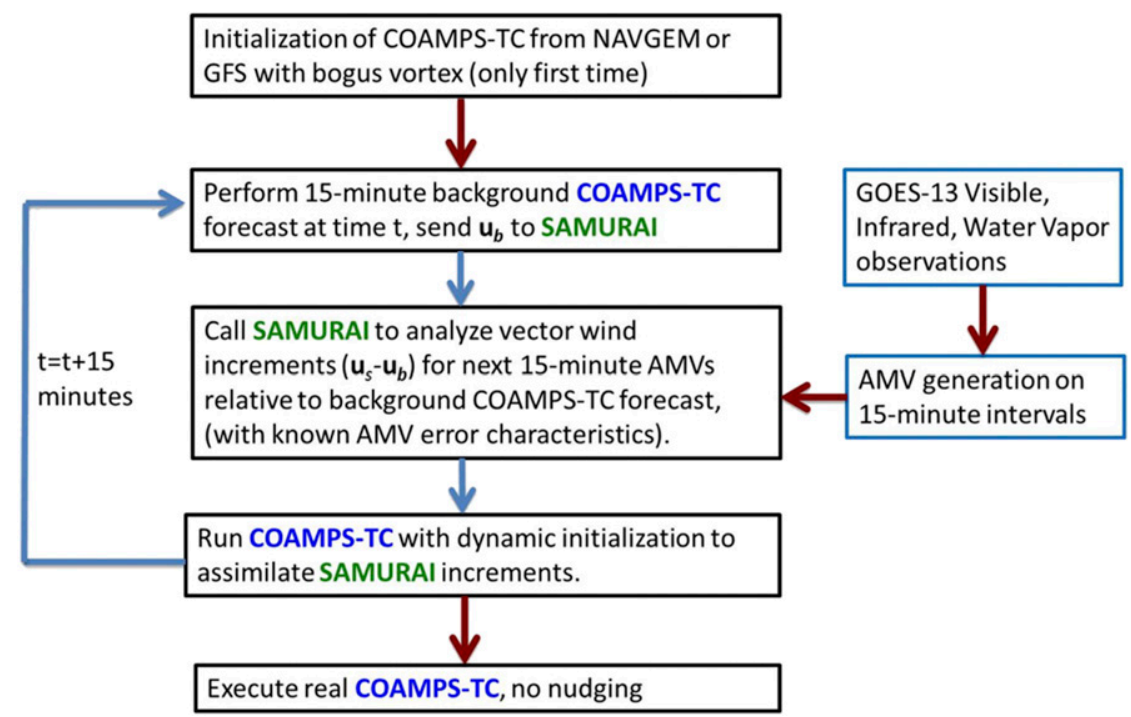

FIG. 3. Flowchart of the SCDI scheme with a cold-start initialization of COAMPS at the first time only, and then 15-min cycling with three steps of creating a background COAMPS-TC forecast, a SAMURAI analysis of vector wind increments, and an integration of COAMPS-TC with nudging of those SAMURAI wind increments in the SCDI that results in initial conditions for a COAMPS-TC forecast (see text).

the next set of AMVs. As described in section 2b, these AMVs and the background COAMPS-TC forecast vector wind $\mathbf{V}_{b}$ components on all three domains are the inputs to the SAMURAI subroutine to create vector wind increments $\left(\mathbf{V}_{s}-\mathbf{V}_{b}\right)$ relative to the background COAMPS-TC forecast. The third step in SCDI is to integrate COAMPS-TC for another $15 \mathrm{~min}$ now including the nudging terms in Eqs. (1) and (2) for these AMV-based SAMURAI wind increments. In this proofof-concept version of SCDI, the background COAMPSTC forecast is utilized rather than each $15-\mathrm{min}$ COAMPS-TC forecast becoming the new background wind fields on the three domains for the next AMV dataset (Fig. 3). At the end of this 15-min cycling throughout the $6 \mathrm{~h}$ to the next synoptic time, the SCDI analysis becomes the initial conditions for the subsequent COAMPS-TC forecast to $72 \mathrm{~h}$ or longer. As will be described later, the advantage of doing this proof-ofconcept demonstration in the Atlantic with 15-min AMVs from GOES-East during TCI-15 is that the HDSS dropwindsondes provide detailed observations to validate the predicted Joaquin vortex structure.

A crucial aspect of the SCDI for assimilating these high temporal and spatial resolution AMVs is the twoway interaction on the three domains of the COAMPSTC model that provides the background forecast wind fields for the SAMURAI analyses of wind increments. As illustrated in the schematic in Fig. 4, the time stepping begins on the $45-\mathrm{km}$ domain 1 with the appropriate SAMURAI wind increments. Then, three time steps are taken on the $15-\mathrm{km}$ domain 2 with the appropriate SAMURAI wind increments. For each of these three domain 2 time steps, three time steps are taken on the $5-\mathrm{km}$ domain 3 centered on the TC forecast position (both domains 2 and 3 are moved with the storm). In each time step on each domain, the mass fields will be adjusted to the SAMURAI wind increment forcing

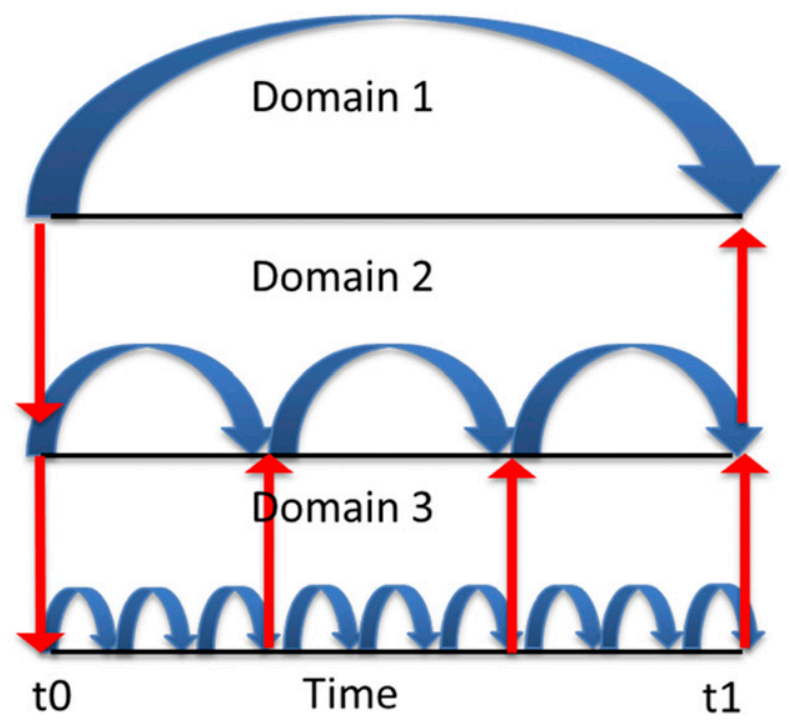

FIG. 4. Schematic of the time steps (blue arrows) on the three domains of the COAMPS-TC with the two-way interaction via the upscale transfer (red arrows) of the solutions from the domain 3 to the domain 2 coincident points and from domain 2 to coincident points within domain 1 . 

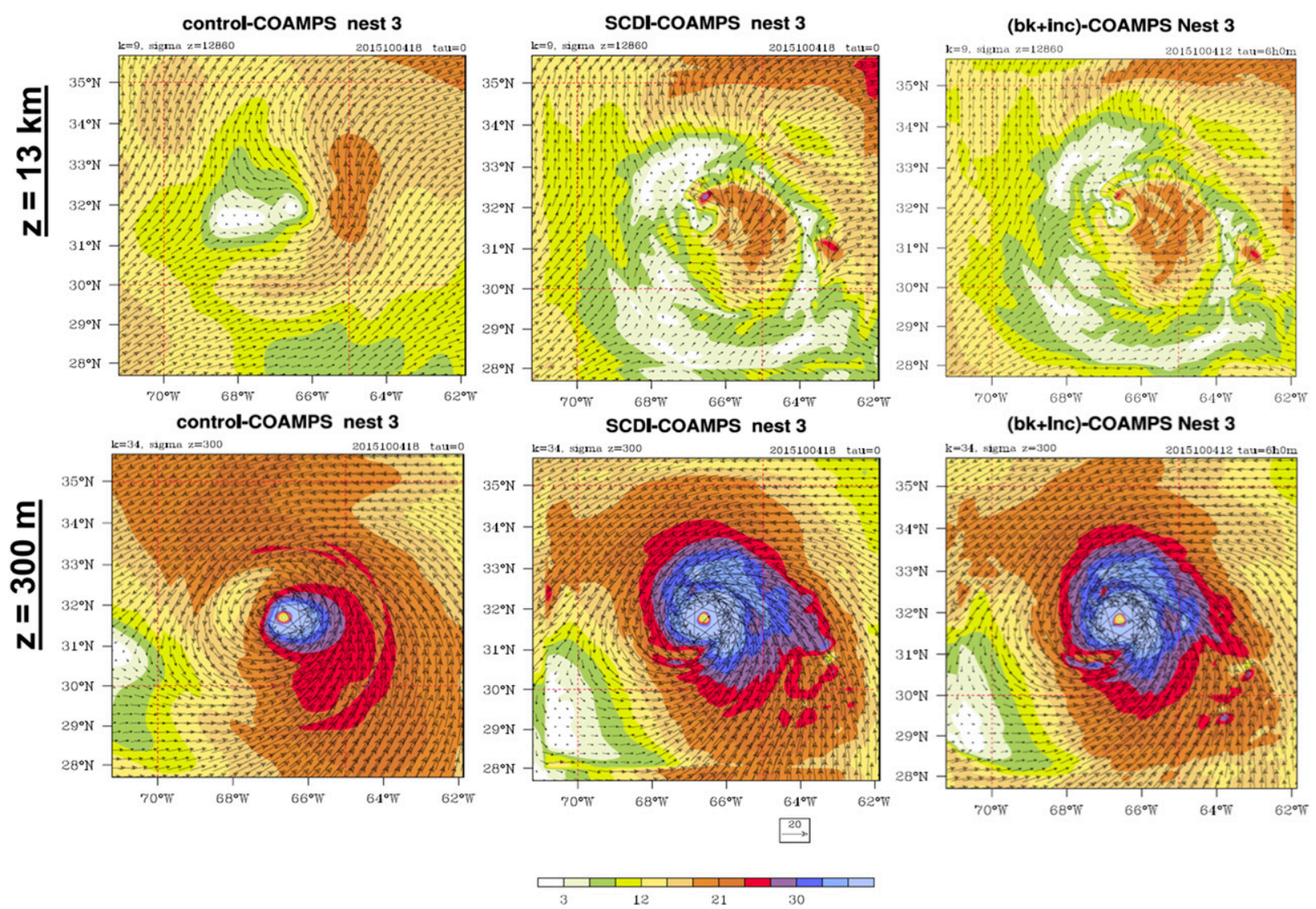

FIG. 5. Wind vectors ( $\mathrm{m} \mathrm{s}^{-1}$; color scale at bottom) on domain 3 at 1800 UTC 4 Oct 2015 at (top) $13 \mathrm{~km}$ and (bottom) $300 \mathrm{~m} \mathrm{for} \mathrm{(left)} \mathrm{the}$ Control COAMPS-TC cold-start initial conditions, (center) SCDI analysis after $6 \mathrm{~h}$ of assimilating a special AMV dataset at 15-min intervals, and (right) COAMPS-TC background plus SAMURAI increments.

derived for that 15-min AMV dataset. Since the time steps for domains 1,2 , and 3 are 90,30 , and $10 \mathrm{~s}$, the SCDI with 15-min AMV datasets will have 10,30, and 90 time steps, respectively, during which the mass fields on these three domains will be adjusted to the AMV-based SAMURAI wind increments during that 15 -min period. Depending on the magnitudes of the wind increments, as in Fig. 2, and their temporal continuity from one AMV 15-min interval to the next, 10 time steps may not be adequate to adjust the mass field to the wind increments in the region of Joaquin on domain 1. Although further testing varying the covariances will be carried out with a larger dataset, the mass does appear to be adjusted to the AMV-based wind increments across domains 2 and 3 based on the early stages of the forecasts in sections 3 and 4 below.

\section{e. Validation of SCDI analyses}

The SCDI analyses at 1800 UTC 4 October will be compared with analyses that are the COAMPS-TC coldstart initial conditions (referred to as Control) as described in section $2 \mathrm{~b}$. The Control $300-\mathrm{m}$ wind analysis in domain 3 centered on Hurricane Joaquin (Fig. 5, bottom left) has a large asymmetry due to the superposition of a symmetric bogus vortex on the background NAVDASAR flow field after removal of the NAVDAS-AR analyzed vortex. Because the NHC best track for Joaquin at 1800 UTC 4 October has a translation speed and direction of $10.9 \mathrm{kt}\left(5.61 \mathrm{~m} \mathrm{~s}^{-1}\right)$ toward the north-northeast (23.8 from north), the maximum wind speeds are in the east-southeast quadrant, and these maximum winds extend much farther to the east-southeast than to the westnorthwest. As indicated at the top of the flowchart in Fig. 3, the SCDI analysis started from a similar cold-start wind field at 1200 UTC 4 October (not shown), but the SCDI has a more symmetric surface wind field for a 85 -kt $\left(1 \mathrm{kt} \approx 0.51 \mathrm{~m} \mathrm{~s}^{-1}\right.$ ) hurricane (Fig. 5 , bottom center). These SCDI 300-m wind speeds and directions are only slightly asymmetric with maximum winds in the southeast quadrant. Furthermore, this band of enhanced wind speeds wraps all the way around the center so that the SCDI analysis has wind speeds of about $25 \mathrm{~m} \mathrm{~s}^{-1}$ near $68^{\circ} \mathrm{W}$ to the west of the center where the Control has a relative minimum of about $12 \mathrm{~m} \mathrm{~s}^{-1}$. In the bottom-right 
panel of Fig. 5 is the vector sum of the background COAMPS-TC wind field plus the SAMURAI increments relative to that background field, which in the outer regions beyond the cirrus canopy should be close to the lower-tropospheric AMV observations. This better agreement in vector directions and speeds with the SCDI analysis than with the Control is particularly noteworthy in the bottom-left corner with light winds and in the bottom-right corner with stronger northward flow.

Even larger differences between the Control (Fig. 5, top left) and the SCDI analysis (top center) are noted in the $13-\mathrm{km}$ wind fields. The Control analysis has a continuous, broad band of strong winds wrapping cyclonically around a small region of minimum wind speeds directly above the $300-\mathrm{m}$ center. By contrast, the SCDI analysis has a concentrated region of cyclonic outflow in the eastern quadrant. However, the more dramatic difference is in the extensive regions of minimum wind speeds in the western semicircle that appear to represent the Joaquin outflow opposing the southwesterly environmental flow in the Control analysis. The vector sum of the background COAMPS-TC wind field plus the SAMURAI increments relative to that background field at $13 \mathrm{~km}$ (top right), which again should be close to the upper-tropospheric AMV observations, is indeed much closer to the SCDI analysis (top center) than to the Control (top left). Although the AMVs in Fig. 1 are at an earlier time, note that the AMVs near the outer edge of the cirrus canopy seem to indicate almost a discontinuity in the upshear direction, which along with the cloud-free region in the infrared images suggests subsidence just beyond the cirrus edge. In summary, the SCDI analysis has been successful in ingesting the AMV observations over and adjacent to Joaquin to provide a depiction of the outflow layer that is markedly different from the Control analysis.

As described in Doyle et al. (2017), the NASA Hurricane Imaging Radiometer (HIRAD) was another instrument deployed on the NASA WB-57 during TCI-15. This four-channel, C-band, synthetic thinned-array radiometer has been developed to measure a $\sim 50-\mathrm{km}$-wide swath of ocean surface winds along the flight path. At wind speeds above $15 \mathrm{~m} \mathrm{~s}^{-1}$, the C-band emissivity increases with increasing surface wind speed due to increasing foam coverage on the ocean surface. Because the four C-band channels also have varying sensitivities to rain, both the surface wind speed and rain rate can be retrieved from the HIRAD observations along the 50-km swath. Cecil and Biswas (2017) have compared the HIRAD surface wind speeds with the HDSS dropwindsonde wind speeds in the various TCI-15 missions, including the four missions in Joaquin (Doyle et al. 2017, their Fig. 6).
The HIRAD surface wind speeds (Fig. 6a) during the east-to-west flight leg with a center overpass around 1900 UTC 4 October can be compared (qualitatively; we do not have access to digital values) with the $10-\mathrm{m}$ wind speeds from the Control (Fig. 5, bottom left) and the SCDI analysis based on $6 \mathrm{~h}$ of AMVs (Fig. 5, bottom center). The outer circumference of the large circular blue region near $31.85^{\circ} \mathrm{N}, 66.5^{\circ} \mathrm{W}$ represents the $15 \mathrm{~m} \mathrm{~s}^{-1}$ isotach within the eye, which is not analyzed in detail since the HIRAD is not sensitive to wind speeds below $15 \mathrm{~m} \mathrm{~s}^{-1}$. The surface wind speeds then sharply increase to $>40 \mathrm{~m} \mathrm{~s}^{-1}$ about $0.1^{\circ}$ latitude to the northeast of this $15 \mathrm{~m} \mathrm{~s}^{-1}$ isotach around the eye. A narrow band of $>35 \mathrm{~m} \mathrm{~s}^{-1}$ wind speeds extends from north of the eye around to the southeast of the eye that may be associated with the eyewall. The orientation and width of this band of strong winds from HIRAD is more consistent with the isotachs from the SCDI analysis (Fig. 5, bottom center) than with the broad band of maximum winds in the Control (Fig. 5, bottom left). Likewise, the northsouth-oriented HIRAD isotach maxima along $67^{\circ}$ and $67.5^{\circ} \mathrm{W}$ is more consistent with the broad isotach spiral band in the SCDI analysis than in the Control.

Creasey and Elsberry (2017) have developed a technique to estimate the zero wind center (ZWC) positions every $200 \mathrm{~m}$ in the vertical from the HDSS dropwindsondes deployed from the WB-57 flying at $60000 \mathrm{ft}$. The Creasey and Elsberry analysis of these ZWCs as the WB-57 overflew the Joaquin center at about 1900 UTC 4 October (Fig. 7a) revealed a relatively small tilt of the vortex from 1.5 to $6.5 \mathrm{~km}$ (lowest five red dots), but then an eastward tilt of about $20 \mathrm{~km}$ was analyzed from 6.5 to $10.5 \mathrm{~km}$ (uppermost five red dots). It is noteworthy that a similar vortex tilt is present in the 1800 UTC SCDI analysis (Fig. 7b). In this east-towest cross section of the SCDI-analyzed tangential winds, the center positions and the adjacent isotachs are more vertically oriented in the lowest $5-6 \mathrm{~km}$, and then the center positions, and the isotachs to the east, have a more pronounced eastward tilt to about $11 \mathrm{~km}$. Above $11 \mathrm{~km}$, the center positions in both the SCDI analysis and the Creasey and Elsberry (2017) ZWCs are not well defined. At 1800 UTC 4 October, the Control vortex structure has a nearly uniform westward tilt with height and amounts to about $20 \mathrm{~km}$ by an elevation of $13 \mathrm{~km}$ (Fig. 7c). This vortex structure is a function of the bogus vortex superposed on the background NAVDAS-AR analysis.

These differences in vortex tilt structure between the Control and the SCDI analyses are related to the innercore temperature anomalies. Hendricks et al. (2018) calculated the temperature anomaly within the eye region relative to the near environment of Joaquin in each 
a)

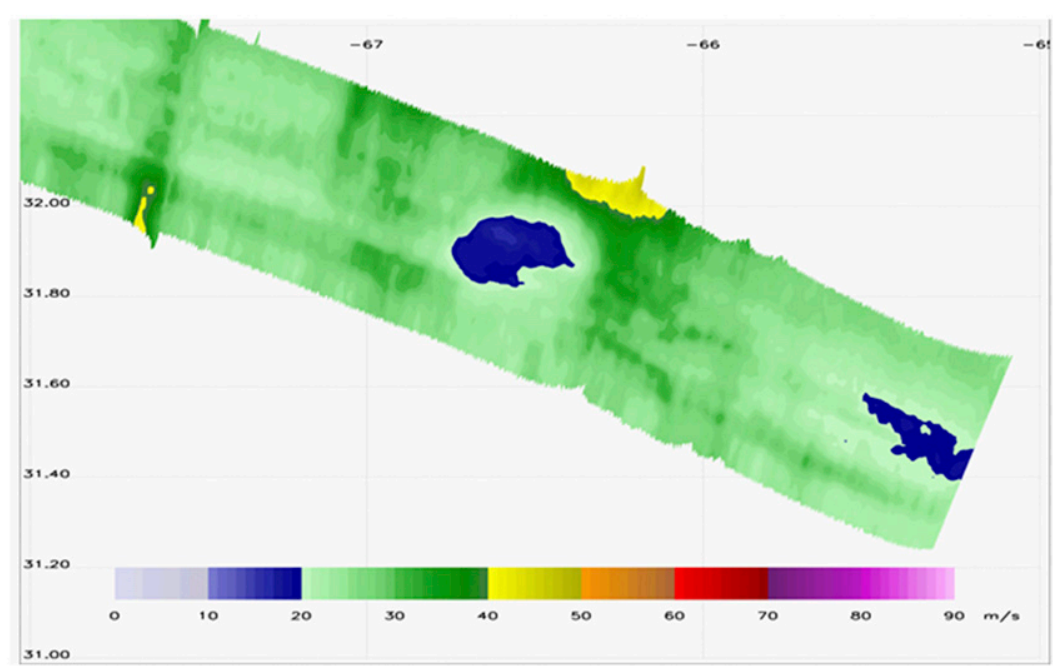

b)

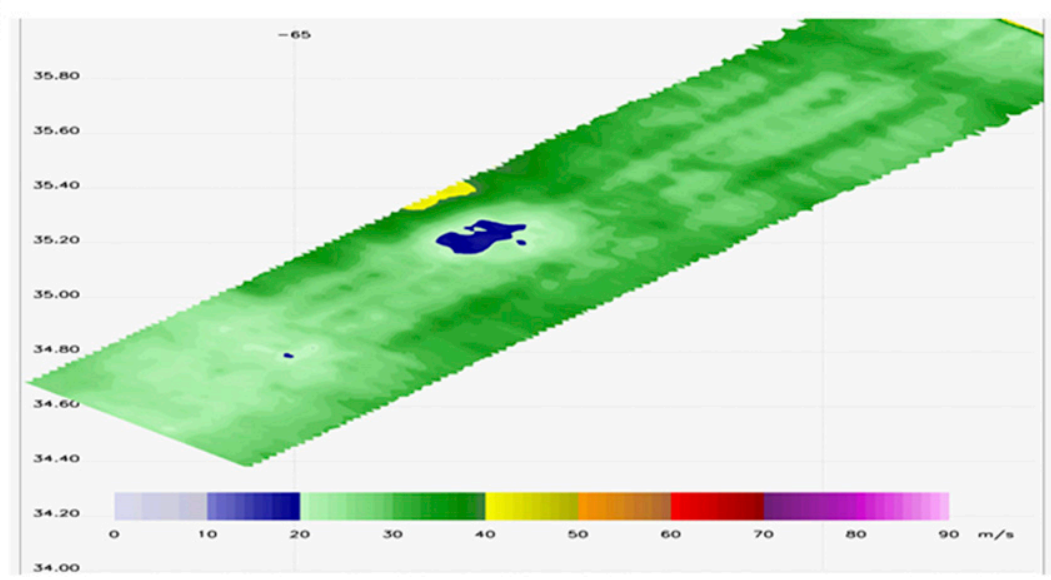

FIG. 6. Surface wind speeds $\left(\mathrm{m} \mathrm{s}^{-1}\right.$; isotach color scale at bottom) from the HIRAD observations as the NASA WB-57 overflew Hurricane Joaquin (a) from east to west around 1900 UTC 4 Oct 2015 and (b) from south to north around 2000 UTC 5 Oct (Image provided by D. Cecil.)

of the four WB-57 missions by averaging the temperature profiles from the HDSS soundings within $20 \mathrm{~km}$ of the center and subtracting the average of the Control temperature values within the $300-400-\mathrm{km}$ annulus about the center. The raw HDSS temperatures were interpolated onto a uniform grid in the vertical direction with a spacing of $50 \mathrm{~m}$. The positive temperature anomaly at 1800 UTC 4 October (Fig. 7d, black line) rapidly increased from near the surface to a maximum value of $7^{\circ} \mathrm{C}$ around $4 \mathrm{~km}$ but rapidly decreased to about $6 \mathrm{~km}$ before then decreasing more slowly up to $8 \mathrm{~km}$. This relatively shallow and weak warm core is from the hydrostatic equation consistent with a weaker cyclonic vortex at the surface, and Joaquin was near the end of the rapid decay period with an intensity of $85 \mathrm{kt}$. Whereas the SCDI analysis at 1800 UTC 4 October
(Fig. 7, green line) has a similar increase in the warm anomaly with height up to $4 \mathrm{~km}$, the temperature anomaly continues to increase to a height of $7 \mathrm{~km}$ rather than having a relatively cool layer between 4 and $10 \mathrm{~km}$ as in the HDSS soundings. By contrast, the Control temperature anomaly is smaller than the HDSS soundings in the $2-5-\mathrm{km}$ layer and then is larger up to a maximum near $7 \mathrm{~km}$. Only the HDSS temperature anomaly vertical structure with a relative maximum at $4 \mathrm{~km}$ has an indication (from gradient thermal wind considerations) of decreasing cyclonic circulation with elevation that might be associated with the vortex tilt structure in Figs. 7a and 7b.

Because of the cold-start procedure in which a bogus vortex wind field to represent the TC is assimilated into the global wind field, the Control COAMPS-TC model 
a)

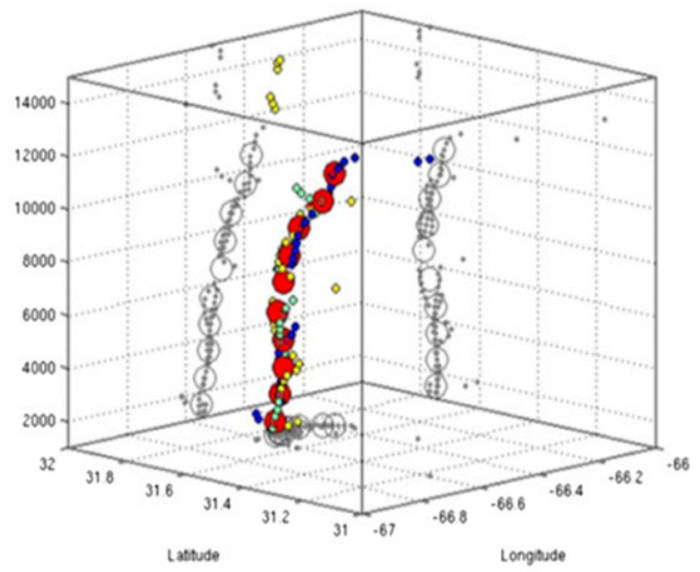

C)

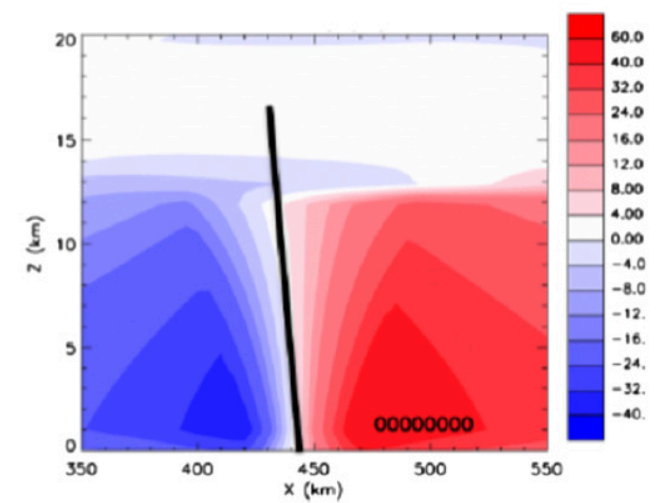

b)

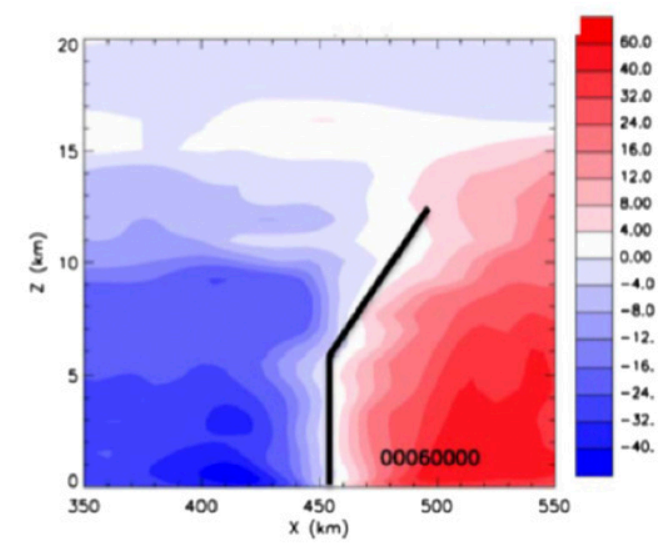

d)

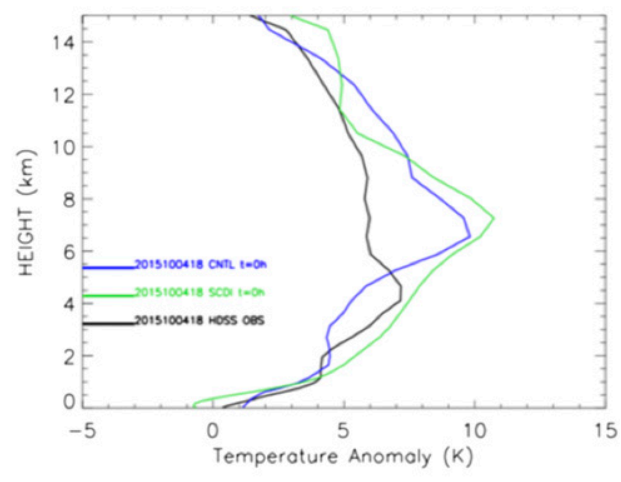

FIG. 7. (a) Hurricane Joaquin vortex tilt at 1900 UTC 4 Oct based on ZWCs at 1-km intervals (large red dots) and at 200-m intervals (small dots) as calculated by Creasey and Elsberry (2017) from a sequence of three HDSS dropwindsondes during a center overpass. (b) SCDI analysis of the tangential wind speeds ( $\mathrm{m} \mathrm{s}^{-1}$; isotach color scale on right) in an east-west cross section through the analyzed center. The heavy black line indicates the vortex tilt inferred from the SCDI analysis. (c) As in (b), but for the Control COAMPS-TC forecast initial conditions at 1800 UTC 4 Oct. (d) Inner-core temperature anomaly relative to the Control COAMPS-TC temperatures within a 300-400-km radius annulus at 1800 UTC 4 Oct for Control (blue line), SCDI analysis (green line), and derived from HDSS dropwindsondes (black line).

at the initial time has no clouds and the model physics associated with the bogus vortex wind field must then spin up the cloud pattern. By contrast, the SCDI analysis already has the associated air-sea enthalpy and momentum fluxes, boundary layer physics, and convective cloud and latent heating distribution that are consistent with the wind (and mass) fields of the TC at the initial time. For example, the simulated radar reflectivity that is associated with the 1800 UTC 4 October SCDI wind analyses in Fig. 5 is given in Fig. 8b. Note that the simulated convective bands around the center are representative of a weak TC. However, the absence of convective bands at outer radii in the southwest quadrant implies subsidence in that quadrant and is consistent with the environmental vertical wind shear that is tilting the vortex as in Figs. 7a and 7b. Unfortunately, the NASA WB-57 did not have a cloud radar, and NOAA WP-3D planes that do have cloud radars were not available, and so no in situ validation of the simulated radar reflectivity pattern in Fig. 8b was possible. However, the microwave satellite image at 1851 UTC 4 October in Fig. 8a does support the banded cloud structure around the center and the absence of deep convection in the southwest quadrant.

The SCDI analysis of $850-\mathrm{mb}$ relative humidity $(\mathrm{RH})$ at 1800 UTC 4 October (Fig. 8d) has a cyclonic banded 


\section{SATELLITE}

a)

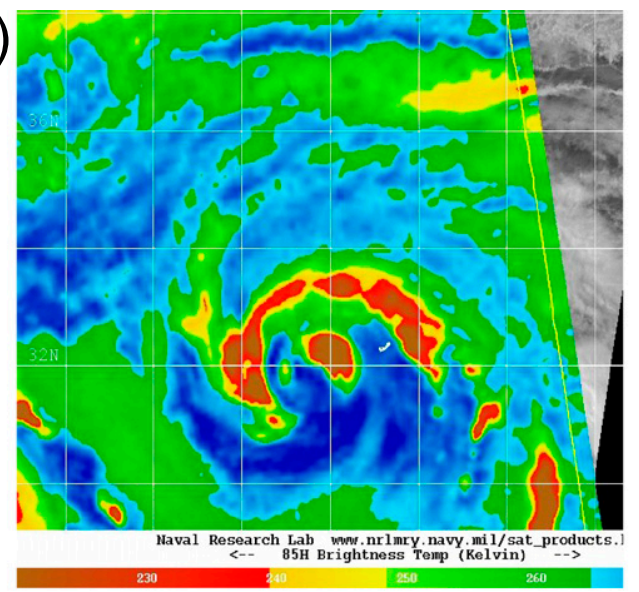

CNTL

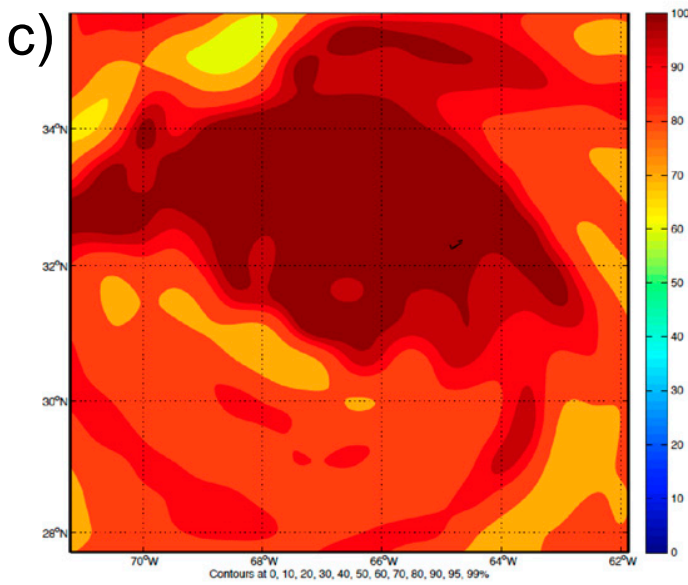

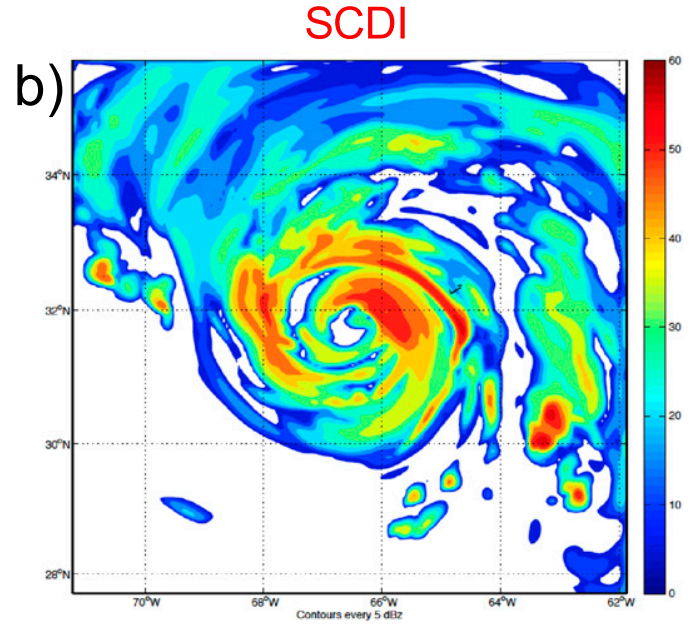

SCDI

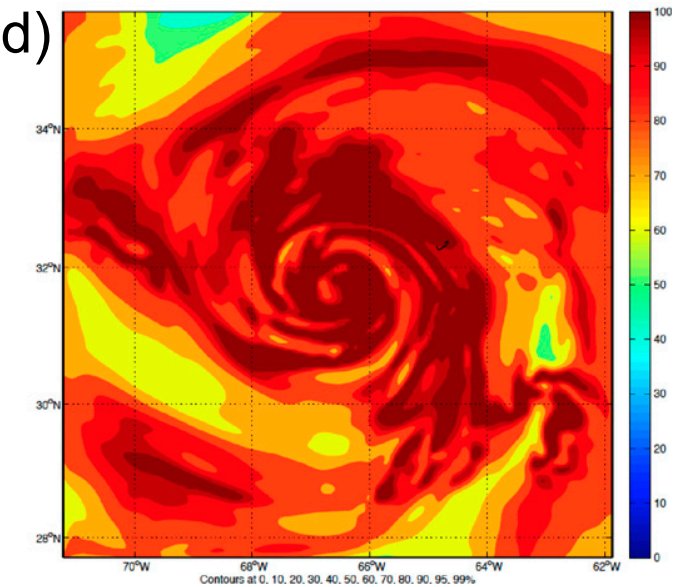

FIG. 8. (a) Microwave satellite image at 1851 UTC 4 Oct 2015 indicating the cloud distribution associated with Hurricane Joaquin. (b) Simulated radar reflectivity (dBZ; color scale on right) associated with the SCDI analysis at 1800 UTC 4 Oct after $6 \mathrm{~h}$ of assimilating a special AMV dataset at 15-min intervals. Relative humidity (\%; color scale on right) at $850 \mathrm{mb}$ across domain 3 at 1800 UTC 4 Oct for the (c) Control cold-start initial conditions and (d) SCDI analysis, as in (b).

structure that is representative of a hurricane and is fully consistent with the simulated radar reflectivity in Fig. 8b. That is, a closed band of $\mathrm{RH}=100 \%$ surrounds the center of Hurricane Joaquin, and the outer RH $=100 \%$ bands spiral into the central region, similar to the simulated radar reflectivity bands. By contrast, the Control 850-mb RH analysis only has a broad circular band around the central region and a continuous region of high $\mathrm{RH}$ in the northern semicircle (Fig. 8c). Thus, the SCDI analysis clearly has a more representative RH distribution of a TC than the Control and is generally consistent with the microwave image in Fig. 8a.

In summary, these special 15-min AMVs from the TCI-15 field experiment have been fully utilized via the SCDI technique because the 15-min SAMURAI analysis of wind increments relative to the background COAMPS-TC forecast has been successfully assimilated via the COAMPS-TC dynamic initialization. A key feature of that dynamic initialization is the mass field can adjust to those AMV-based wind increments during the 30 and 90 time steps on domains 2 and 3 during each 15-min AMV dataset. The COAMPS-TC model physics then predicts the convection pattern consistent with those wind and mass fields each $15 \mathrm{~min}$. While only preliminary comparisons have been made thus far with the special TCI-15 datasets, the similarity of the vortex tilt from the SCDI analysis with that revealed by the Creasey and Elsberry (2017) analysis of the ZWCs from the HDSS dropwindsondes is very encouraging. 


\section{Example of SCDI impacts on regional model forecasts}

Velden et al. (2017) and Kim et al. (2017) summarize many studies demonstrating the positive impacts that hourly AMVs have had on tropical cyclone track forecasts. In most of these studies, the AMV impact has been demonstrated with global models, which may provide more accurate track forecasts than do the regional numerical models. For the three Atlantic hurricanes that Velden et al. (2017) studied, the operational HWRF track forecasts were already quite accurate and thus the impact of the enhanced hourly AMVs was small.

For the Joaquin track forecasts from the 1800 UTC 4 October 2015 case described in section 2, the challenge was to forecast the accelerated movement as Joaquin was recurving into the midlatitudes. For the operational COAMPS-TC initiated from NAVGEM initial and boundary conditions (labeled as CNTL-NAVGEM) track forecast (Fig. 9a), a left-of-track bias leads to increasing 24-, 48-, and 72-h errors of 59, 147, and $290 \mathrm{~km}$. By contrast, the COAMPS-TC forecast initiated from the1800 UTC 4 October SCDI analysis (labeled SCDINAVGEM) in domains 2 and 3 first has a right-of-track bias with 24- and 48-h errors of $38 \mathrm{~km}$ and $117 \mathrm{~km}$, respectively, and then is nearly coincident with the $72-\mathrm{h}$ position with only a $55-\mathrm{km}$ track error. A second version of COAMPS-TC initiated from NCEP Global Forecast System (GFS) initial and boundary conditions (labeled as CNTL-GFS) track forecast has a right-of-track bias with 24-, 48-, and 72-h errors of 51, 100, and $95 \mathrm{~km}$, respectively (Fig. 10a). Although the corresponding COAMPS-TC forecast with the 1800 UTC 4 October SCDI analysis as initial conditions (labeled SCDI-GFS) has similar track forecast errors at 24 and $48 \mathrm{~h}$ (64 and $84 \mathrm{~km})$, the $72-\mathrm{h}$ error is only $35 \mathrm{~km}$, which is a $60-\mathrm{km}$ smaller error than the CNTL-GFS.

As noted above, these very accurate COAMPS-TC track forecasts from the SCDI analysis were achieved when only domains 2 and 3 were utilized. When the 45-km domain 1 SCDI analysis, which was forced only by the AMV-based SAMURAI wind increments relative to the background COAMPS-TC forecast, was also included, the COAMPS-TC track forecasts were not improved (not shown). The implication is that the global model data assimilation, which for NAVGEM has a 31-km horizontal resolution, and also assimilates conventional observations (especially over the United States just to the west of Joaquin), has a better representation of the large-scale flow that is critical for TC track forecasting. While this result will be checked with a large sample of track forecasts, the present plan is to only utilize the SCDI analyses on the inner two grids that move with the $\mathrm{TC}$ as initial conditions for the COAMPS-TC forecast step at the bottom of the flowchart in Fig. 3.

The primary benefit of the regional numerical models is in forecasting the TC intensity. The intensity $\left(V_{\max }\right)$ forecast challenge at 1800 UTC 4 October is to predict a continued rapid decay to $75 \mathrm{kt}$ at 0000 UTC 5 October and then a constant $V_{\max }$ until 0000 UTC 6 October according to the NHC best track file (Figs. $9 \mathrm{~b}$ and $10 \mathrm{~b}$, black lines). However, the minimum sea level pressure (MSLP; Figs. 9c and 10c) in the NHC best track file continues to rise beyond 0000 UTC 5 October and then has only a 12-h period of constant MSLP before again rapidly rising, which appears to be inconsistent with the 30-h period of constant $V_{\max }$ in the NHC best track (Figs. 9b and 10b).

The CNTL-NAVGEM cold-start COAMPS-TC forecast with a bogus vortex continues to rapidly decay Joaquin to $60 \mathrm{kt}$ at 0600 UTC 5 October and then predicts a quasi-constant intensity for $18 \mathrm{~h}$ before intensifying to $75 \mathrm{kt}$ at 1200 UTC 6 October, and then has an accurate prediction of the subsequent decay of Joaquin (Fig. 9b, blue line). The COAMPS-TC forecast with SCDI-NAVGEM initial conditions analysis replacing the bogus vortex within domains 2 and 3 (Fig. 9b, green line) predicts a longer rapid decrease in $V_{\max }$, a more irregular period of constant intensity, and a similar brief intensification to $75 \mathrm{kt}$ before the subsequent decay.

The CNTL-GFS intensity $\left(V_{\max }\right)$ forecast has a too slow decrease in $V_{\max }$ the first $6 \mathrm{~h}$ and then underforecasts $V_{\max }$ during the period that the NHC best track has a constant $V_{\max }=75 \mathrm{kt}$ (Fig. 10b, blue line). The corresponding COAMPS-TC forecast with the SCDIGFS initial conditions continues the rapid decrease in $V_{\max } 18 \mathrm{~h}$ beyond when the NHC best track begins that constant $V_{\max }$, and then after reintensifying this SCDIbased forecast verifies within about $5 \mathrm{kt}$ for the remainder of the constant intensity period of Joaquin (Fig. 10b, green line).

Thus, one might consider the Control and the SCDIbased COAMPS-TC intensity $\left(V_{\max }\right)$ forecasts for both the NAVGEM (Fig. 9b) and the GFS (Fig. 10b) initial and boundary conditions to be rather deficient. However, the intensity (MSLP) forecasts in Figs. 9c and 10c, respectively, agree more closely with the NHC MSLPs for this period of Joaquin. Both the CNTL-NAVGEM and SCDI-NAVGEM COAMPS-TC forecasts in Fig. 9c begin with slightly lower pressures, but from day 2 (0000 UTC 5 October) to day 3 the MSLPs are quite similar to the NHC best track values. The corresponding CNTL-GFS COAMPS-TC forecast (Fig. 10c, blue line) not only starts with a low MSLP, but has persistently lower MSLPs relative to the NHC values throughout the 
a)

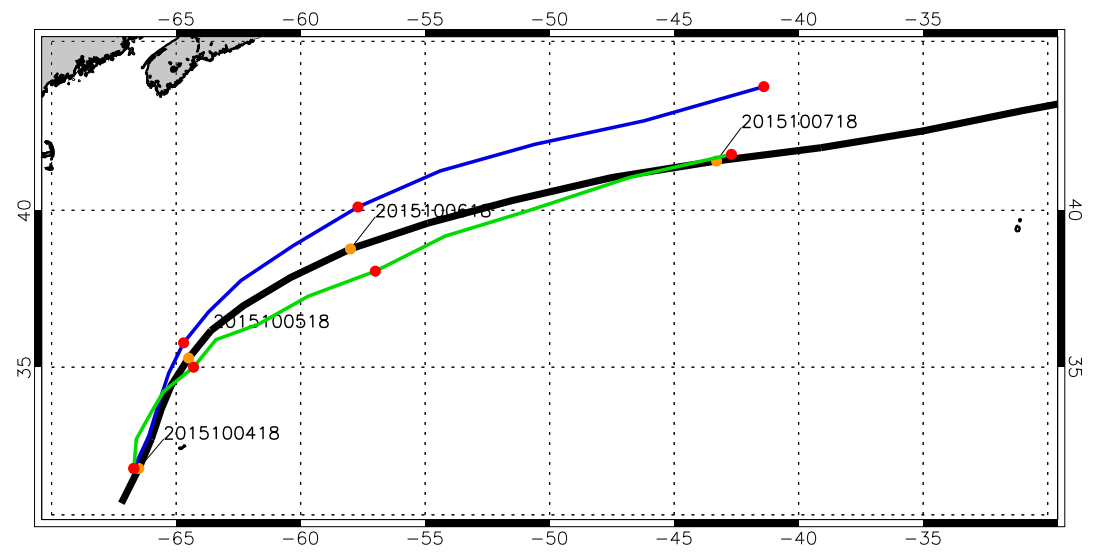

b)

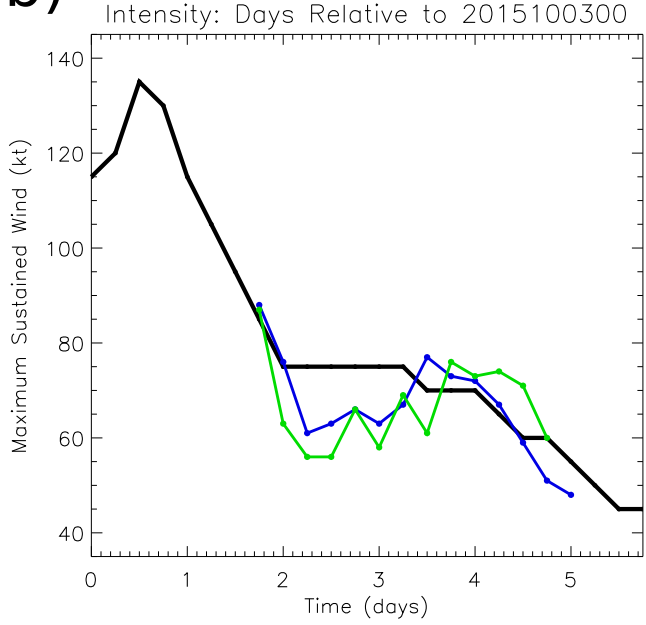

c)

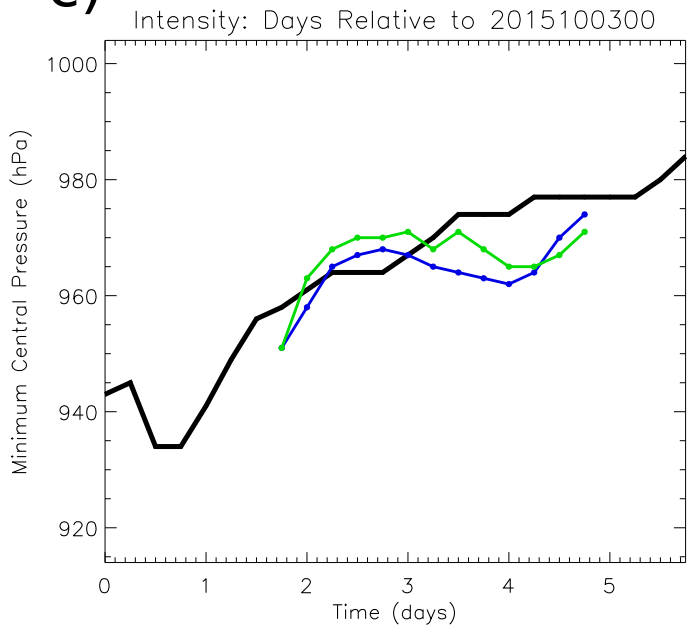

FIG. 9. (a) Track forecast verification relative to the NHC best track file for Hurricane Joaquin (black line) beginning at 1800 UTC 4 Oct for the CNTL-NAVGEM COAMPS-TC forecast (blue line) and SCDI-NAVGEM COAMPS-TC forecast (green line) with dots indicating 24-, 48-, and 72-h positions. (b) Intensity ( $V_{\text {max }}$; kt) forecast verification relative to NHC best track file (black line) where day 0 is 0000 UTC 3 Oct and the CNTL-NAVGEM forecast (blue line) and SCDI-NAVGEM forecast (green line) begin at 1800 UTC 4 Oct. (c) Intensity (minimum central pressure; $\mathrm{hPa}$ ) forecast verification as in (b).

forecast. By contrast, the SCDI-GFS COAMPS-TC has an excellent MSLP forecast from day 2 to day 3.5 (Fig. 10c, green line). Given these rather accurate MSLP forecasts, C. Velden (2017, personal communication) simply notes that satellite-based intensity $\left(V_{\max }\right)$ estimates can be found that also continue the rapid decay beyond 0000 UTC 5 October with a subsequent reintensification more similar to the COAMPS-TC forecasts in Figs. 9b and $10 \mathrm{~b}$ rather than the abrupt transition to constant $V_{\max }$ values in the NHC best track.

The 24-h COAMPS-TC forecasts of 10-m wind speed from the CNTL-GFS initial conditions and from the
SCDI-GFS initial conditions at 1800 UTC 4 October are displayed in Figs. 11a and 11b. The corresponding HIRAD surface wind speeds for validation of these 24-h predictions near Joaquin's center are only available along a single south-to-north overpass (Fig. 6b). Although the Control COAMPS-TC forecast starts from $10-\mathrm{m}$ wind speeds at 1800 UTC 4 October that are asymmetric with larger speeds extending far to the southeast of the center, a much more concentric wind field around the center is predicted after $24 \mathrm{~h}$ (Fig. 11a). However, the maximum winds in this Control forecast are in the eastern semicircle, and the $>40 \mathrm{~m} \mathrm{~s}^{-1}$ maximum in the HIRAD 
a)

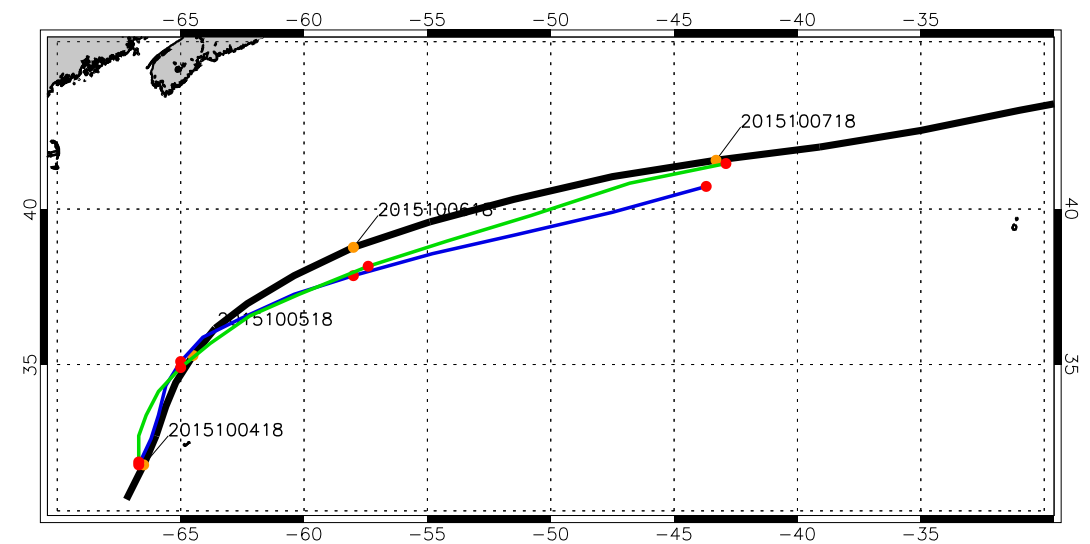

b)

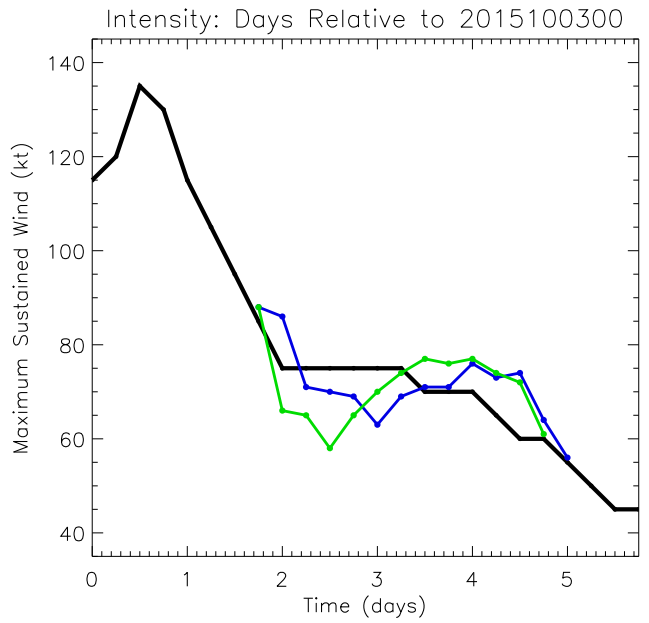

C)

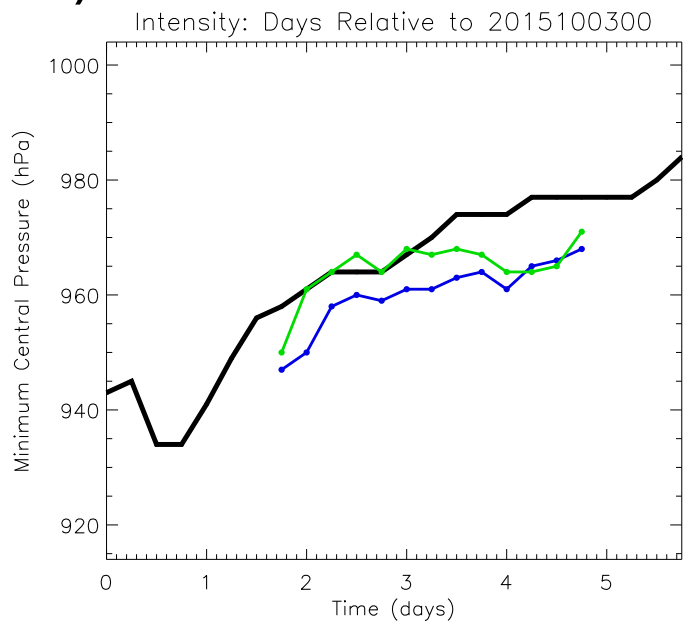

FIG. 10. As in Fig. 9, but for CNTL-GFS and SCDI-GFS COAMPS-TC forecasts.

observations (Fig. 6b) is to the north-northwest of the center. Similarly, the SCDI-initialized COAMPS-TC forecast (Fig. 11b) has the largest 10-m wind speeds just to the east of the center rather than to the north-northwest of the center as in the HIRAD observations. The forecast based on the SCDI analysis has slightly weaker winds $\left(33 \mathrm{~m} \mathrm{~s}^{-1}\right)$ than in the Control forecast $\left(36 \mathrm{~m} \mathrm{~s}^{-1}\right)$, which is consistent with the intensity $\left(V_{\max }\right)$ comparison in Fig. 10b. While $>40 \mathrm{~m} \mathrm{~s}^{-1}$ speeds are indicated in Fig. 6b, Cecil and Biswas (2017) found that the HIRAD surface wind speeds were often larger than the HDSS dropwindsonde near-surface wind speeds. Although the SCDI-based COAMPS-TC forecast may have somewhat underestimated the intensity at 1800 UTC 5 October, an alternate explanation is that the HIRAD had detected a mesoscale circulation that was not representative of the Joaquin inner-core circulation.

\section{Example of SCDI analysis impact on global model forecast}

a. Upscaling the SCDI analysis to global model grid

The NAVGEM utilizes a synthetic TC vortex, and the objective in this section is to upscale the domain 2 SCDI analysis to the NAVGEM grid as a replacement for that synthetic vortex and its environment. The NAVGEM prediction cycle is a short-term $(6 \mathrm{~h})$ forecast starting from $t-6 \mathrm{~h}$ that becomes the background for the NAVDAS-AR (Xu et al. 2005) in which observations over $\pm 3 \mathrm{~h}$ are merged with that background forecast to generate the $t=0 \mathrm{~h}$ global analysis. This NAVDAS-AR analysis then becomes the initial conditions for the next NAVGEM forecast, except when there is a named storm a synthetic TC vortex is inserted after removing all wavenumbers greater than 20 in the original spectral 
analysis, which eliminates short-wavelength features such as TC circulations. An axisymmetric vortex is generated assuming a Rankine vortex profile in which the radius of the maximum winds (RMW) and the exponential decay factor of the radial profile are fixed at $50 \mathrm{~km}$ and 0.6 , respectively, and the $V_{\max }$ and the storm position are provided each $6 \mathrm{~h}$ by the TC warning centers.

Vertical profiles of tangential (but not radial) wind components to represent this synthetic TC vortex are constructed along a total of 12 radial legs at $60-\mathrm{km}$ intervals out to a $600-\mathrm{km}$ radius, and added to the observational database before the 4DVAR data assimilation. It is noted that this synthetic vortex extends only to $400 \mathrm{hPa}$, and thus the TC outflow layer is not included in the initial conditions for NAVGEM forecasts. While the mass field associated with the synthetic vortex wind field is derived from a balance equation solution, there are no pressure perturbations or a warm core to represent the TC above $400 \mathrm{hPa}$. Furthermore, there is no TC secondary circulation of low-level inflow, vertical ascent near the center, and outflow aloft in these initial conditions.

The first test of upscaling the SCDI analysis to the NAVGEM grid utilizes the blending procedure from Herrera et al. (2018), who are developing a unified 4DVAR data assimilation for NAVGEM that will upscale higher-resolution analyses from multiple COAMPS domains. Although Herrera et al. allow for blending coefficients between $\alpha=0$ (NAVGEM fields only) and $\alpha=1$ (COAMPS field only), in this first test $\alpha=1$ is specified so that the full domain 2 SCDI analysis is being projected onto the NAVGEM grid points (not in spectral space). However, $\alpha$ is tapered to zero at the lateral boundaries of the COAMPS-TC domain 2 and at the top of the COAMPS-TC model atmosphere. A key step in the procedure is to vertically interpolate the SCDI fields to the hybrid sigma-pressure coordinate of the NAVGEM. After this blending, no further data assimilation or mass balancing is utilized in this first test.

\section{b. Example of an upscaled SCDI analysis to NAVGEM}

In this demonstration of the upscaling concept, the domain 2 SCDI analysis at 1800 UTC 4 October of the three-dimensional fields of wind, pressure, temperature, and moisture are upscaled to the NAVGEM grid. As an example, the SCDI sea level pressures (SLPs) on the $15-\mathrm{km}$ grid are shown in the middle panel of Fig. 12. Upscaling that SLP field to the $\sim 35$-km NAVGEM grid is shown in the right panel of Fig. 12, and there are only subtle differences in pressure gradients near the center of Joaquin and along the boundaries due to the blending function. Note that the center position in the SCDINAVGEM analysis is very close to that in the SCDI analysis. However, that center position is $0.8^{\circ}$ latitude to the south and $0.8^{\circ}$ longitude to the west of the center position in the CNTL-NAVGEM analysis (Fig. 12, left), which is due to the NAVGEM warm start with the insertion of a synthetic bogus that is quite close to the warning position. Thus, a second step in the blending procedure is to shift the full domain 2 grid such that Joaquin's center is also near the warning position at 1800 UTC 4 October. The tapering of the blending coefficient $\alpha$ to zero at the boundaries adjusts for any spurious wind gradients from this grid shift.

East-west cross sections through the center of the $v$ component of the wind for the CNTL-NAVGEM and the SCDI-NAVGEM vortices at 1800 UTC 4 October are shown in Figs. 13a and 13b, respectively. The inner core of the vortex in the SCDI-NAVGEM initial conditions (Fig. 13b) is more compact at the top of the boundary layer with a radius of maximum wind about $30 \%$ smaller than in the CNTL-NAVGEM (Fig. 13a). Another difference is that the SCDI-NAVGEM vortex does not extend to as high an elevation as in the CNTLNAVGEM case. The northerly wind branch on the west side of Joaquin's vortex in the SCDI-NAVGEM analysis is not as deep because the extension of the southwesterly jet downstream of the trough over Florida is stronger, and the southerly flow branch on the east side is not as deep because of the northerly jet to the east that extends westward over that branch of the vortex. While the difference in the southwesterly flow at $150 \mathrm{hPa}$ between the Control (Fig. 13c) and the SCDI-based analysis (Fig. 13d) is due to a rather subtle wind direction difference, the smaller speeds in the northerly flow in the SCDI-based analysis are quite notable. Recall from Fig. 2 that the AMV-based wind increments indicated that the COAMPS-TC background had too large of a northerly flow in this region to the east of the center. Hendricks et al. (2018) have documented that at 1800 UTC 4 October Joaquin was between large vertical wind shear (VWS) to the north and near-zero VWS to the south. At least at $150 \mathrm{hPa}$, this SCDI-based analysis has strong southwesterly flow to the north with near-zero winds to the south, which is consistent with the VWS distribution in Hendricks et al. (2018).

The advantage of the upscaling of the full threedimensional SCDI analyses of winds, pressures, temperatures, and humidities is that over the COAMPS-TC domain 2 these are balanced mass-wind fields, and include the irrotational (divergent) wind components and vertical motions that constitute the secondary circulation of low-level inflow, ascent in the eyewall, and outflow aloft. As demonstrated in Fig. 8b, the SCDI provides 


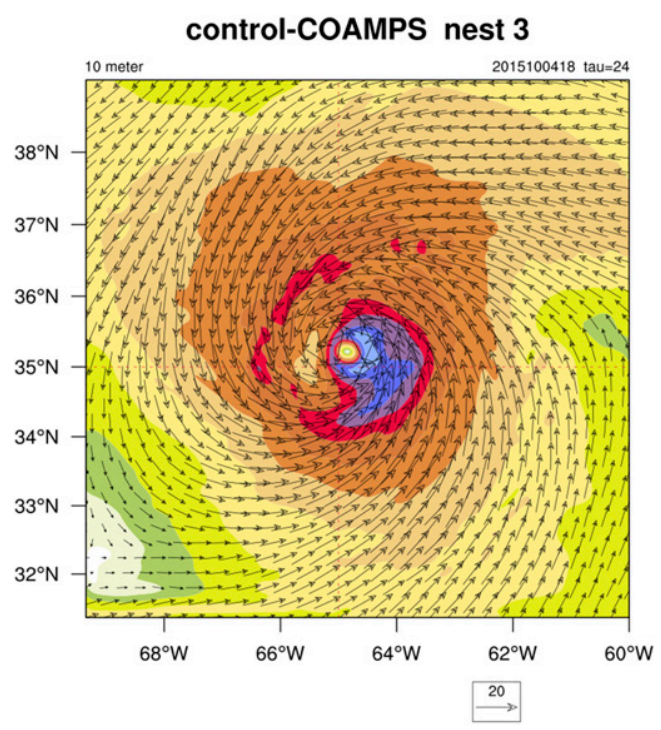

\begin{tabular}{l|l|l|l|l|l|l|l|l|l|}
$\mid$ & $\mid$ & $\mid$ & $\mid$ & $\mid$ & $\mid$
\end{tabular}

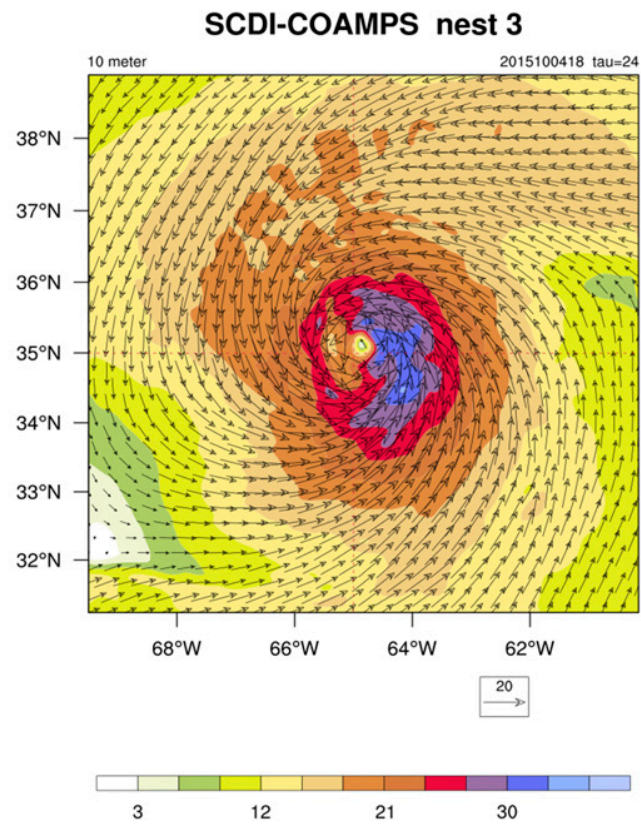

2

30

FIG. 11. Forecasts of 10-m wind speed ( $\mathrm{m} \mathrm{s}^{-1}$; color scale below) at 1800 UTC 5 Oct 2015 across domain 3 of the COAMPS-TC model with initial conditions from the (left) Control-GFS and (right) SCDI-GFS analyses after $6 \mathrm{~h}$ of assimilating a special AMV dataset at 15 -min intervals.

a realistic cloud structure of a $\mathrm{TC}$, and the temperature (Fig. 7d) and relative humidity (Fig. 8d) fields in the SCDI are consistent with that cloud distribution. While the upscaling of the SCDI analyses to the NAVGEM have resulted in a broader and less intense storm because of the more coarse resolution of the NAVGEM, effectively a dynamic initialization has been provided for NAVGEM so that the intensity forecast should evolve smoothly from those initial conditions.

The disadvantages of the Control-NAVGEM with the synthetic TC vortex relative to the SCDI initial conditions are first that the vortex has a broader inner core and offers a less realistic representation of the vortex structure above $400 \mathrm{hPa}$, especially the outflow structure. Even though a balance equation is solved to generate a mass field corresponding to that wind field, the frictional inflow and the ascent near the RMW are representative of the warm-start initial conditions and, thus, may be inconsistent with the synthetic vortex wind field that has been shifted to be closer to the warning position. Since the RMW is at too large of a radius (e.g., Fig. 13a), that frictional inflow and ascent will also be at
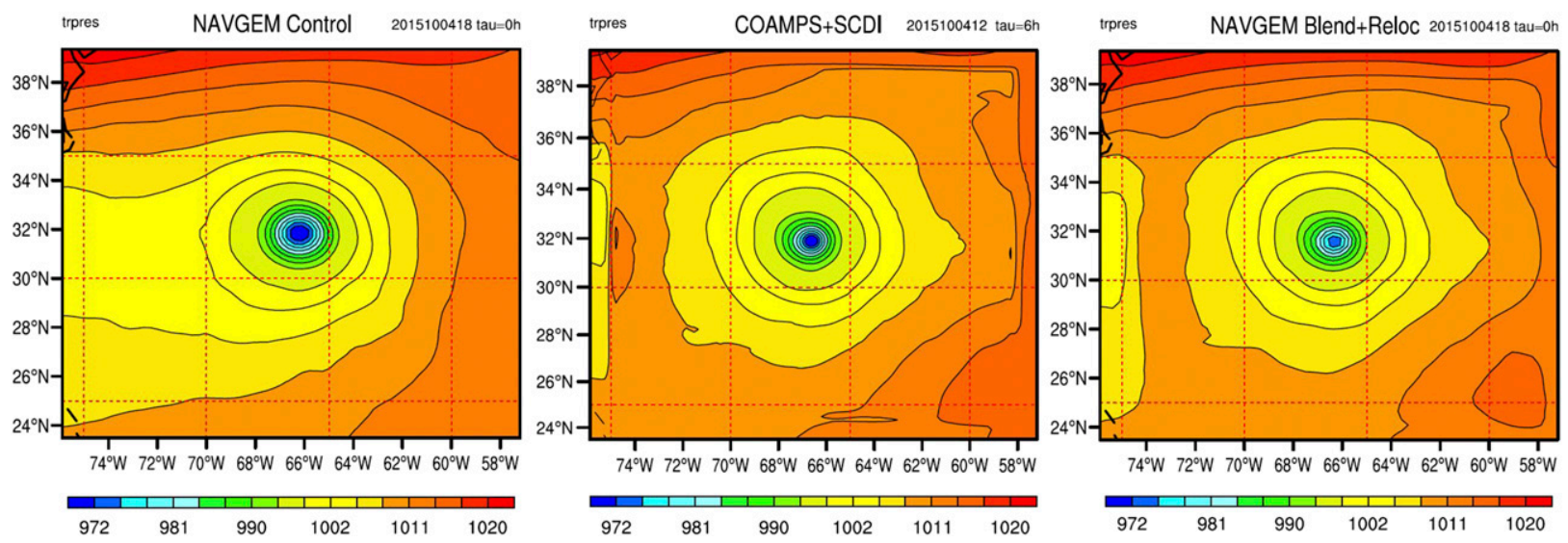

FIG. 12. Sea level pressure (hPa; color scale at bottom) fields across domain 2 at 1800 UTC 4 Oct for the (left) Control-NAVGEM, (center) SCDI analysis, and (right) SCDI-NAVGEM after blending the SCDI analysis into the original NAVGEM initial conditions with a blending coefficient of 1.0 . 


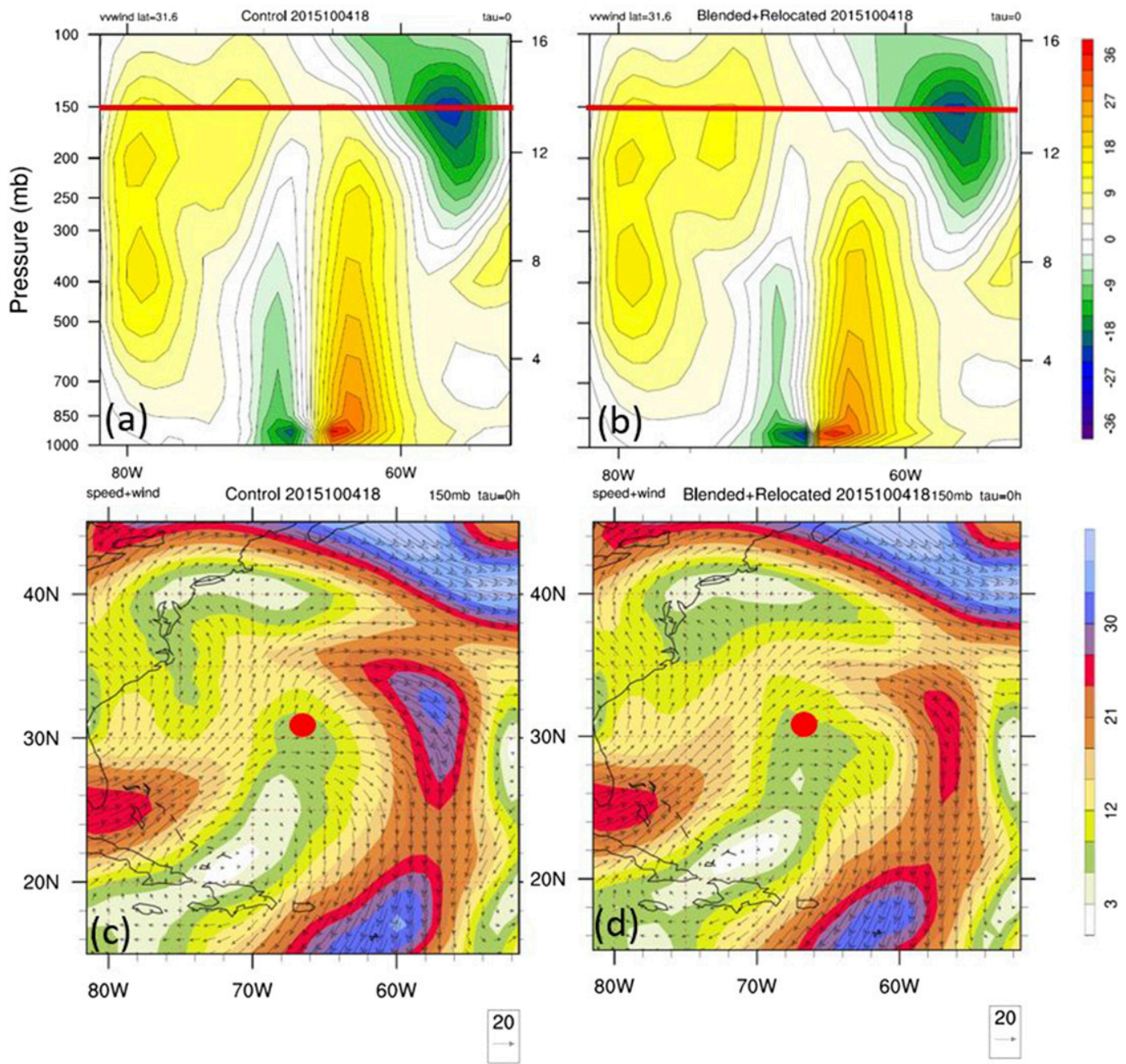

FIG. 13. West-to-east vertical cross sections along $31.6^{\circ}$ latitude of meridional wind components at 1800 UTC 4 October for the (a) CNTL-NAVGEM synthetic vortex and (b) SCDI analysis upscaled into the NAVGEM initial conditions. (c),(d) The 150-hPa wind fields $\left(\mathrm{m} \mathrm{s}^{-1}\right.$; color scale on right), which correspond to (a) and (b), respectively, as indicated by the red lines in those panels. The position of Joaquin is indicated by the red dot.

too large a radius, and the associated diabatic heating in the forecast will tend to lower the surface pressure at that larger radius, which will weaken the pressure gradient at the center and thus decrease the intensity $\left(V_{\max }\right)$ for several hours. Only after the heating inside the RMW leads to a contraction and more realistic storm structure with a smaller RMW will the intensity begin to increase.

\section{c. Impacts on track and intensity forecasts}

Shortly after the 1800 UTC 4 October mission the translation speed of Joaquin began to increase, and Joaquin recurved into the midlatitude westerlies (Fig. 14a, blue line). The SCDI-NAVGEM track forecast (green line) closely agrees with the NHC best track for the first $48 \mathrm{~h}$, but then predicts a more eastward track over the next
$24 \mathrm{~h}$. The CNTL-NAVGEM track forecast (red line) was slightly faster and deviated to the west of the NHC best track during the first $48 \mathrm{~h}$ and, then (perhaps coincidently), agreed very well with the best track through $72 \mathrm{~h}$.

As with the Control and SCDI-based COAMPS-TC intensity forecasts (Figs. 9 and 10), the SCDI-NAVGEM intensity $\left(V_{\max }\right)$ forecast continued the rapid decay of Joaquin beyond 0000 UTC 5 October (Fig. 14b; $t+6 \mathrm{~h}$ ), but then predicted a near-constant $V_{\max }$ for the next $24 \mathrm{~h}$ that is very similar to the best track intensities. However, the SCDI-NAVGEM forecast then intensified Joaquin to $50 \mathrm{~m} \mathrm{~s}^{-1}$ in the next $18 \mathrm{~h}$ rather than slowly decaying. The CNTL-NAVGEM intensity forecast began with a smaller intensity, decayed by a smaller amount during the first $6 \mathrm{~h}$, and as for the SCDI-NAVGEM forecast 

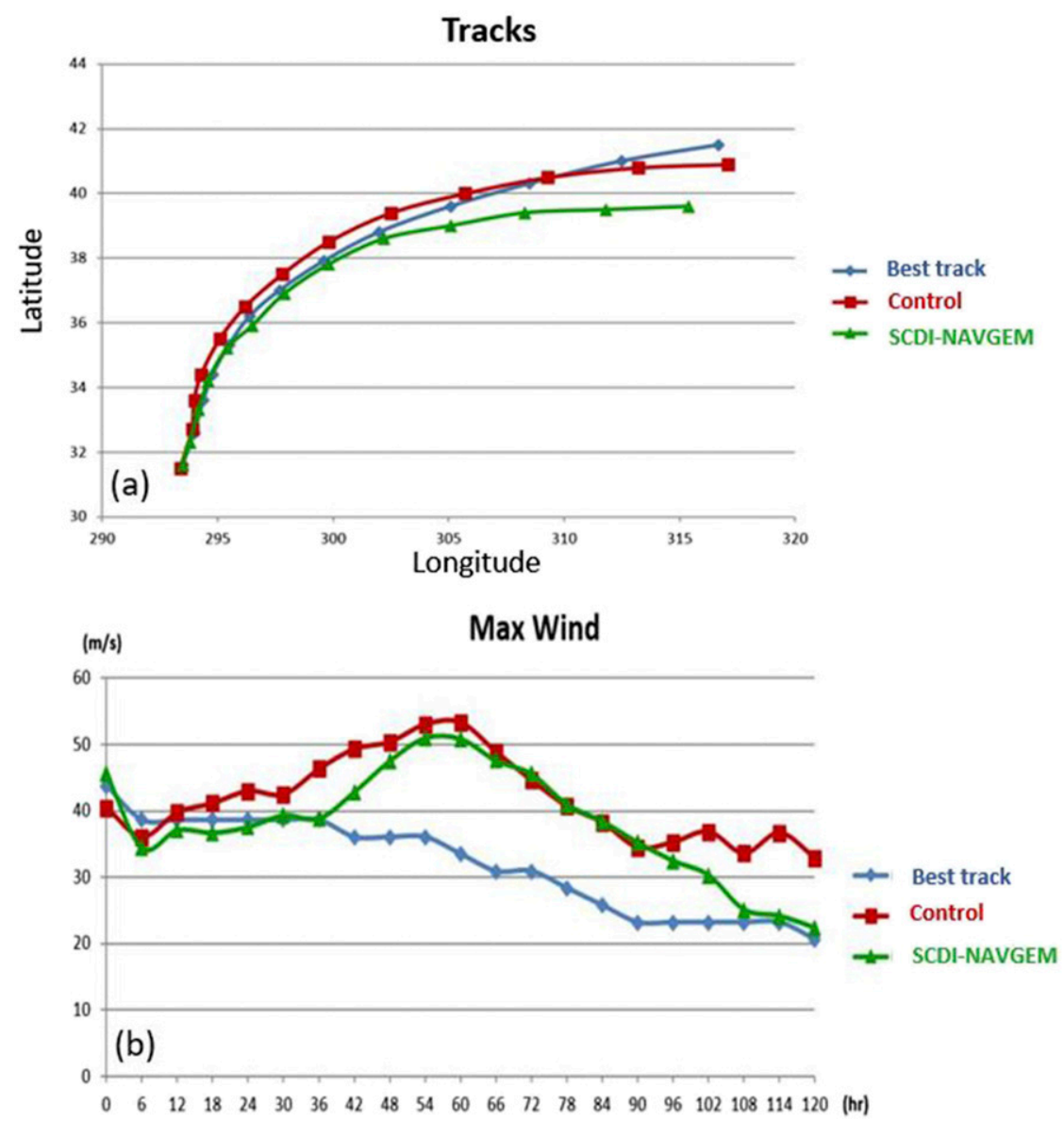

FIG. 14. (a) Track forecasts from $1800 \mathrm{UTC} 4$ Oct to $72 \mathrm{~h}$ with symbols each $6 \mathrm{~h}$ relative to the NHC best track (blue line) of Joaquin by CNTL-NAVGEM (red line) and SCDI-NAVGEM (green line). (b) As in (a), but for intensity $\left(V_{\max } ; \mathrm{m} \mathrm{s}^{-1}\right)$.

predicted a near-constant intensity for $24 \mathrm{~h}$ before intensifying to $50 \mathrm{~m} \mathrm{~s}^{-1}$ rather than decaying.

The 24-h forecasts from 1800 UTC 4 October of the 10 -m wind fields on the domain 2 grid for the CNTLNAVGEM and the SCDI-NAVGEM analyses are shown in Figs. 15a and 15b, respectively. The differences between these NAVGEM forecasts are similar to the differences between the 24-h COAMPS-TC forecasts in Figs. 11a and 11b. That is, both Control forecasts have broader regions of $20 \mathrm{~m} \mathrm{~s}^{-1}$ winds in the northwest quadrant, and the maximum winds wrap around the center. By contrast, the SCDI-based forecasts have a more compact vortex circulation, and specifically the gale-force winds (e.g., $18 \mathrm{~m} \mathrm{~s}^{-1}$ in Fig. 15) along $36^{\circ} \mathrm{N}$ extend from $62^{\circ}$ to $66.5^{\circ} \mathrm{W}$ versus from $61.5^{\circ}$ to $68^{\circ} \mathrm{W}$ for CNTL-NAVGEM. The SCDI-NAVGEM forecast has a smaller region of maximum winds to the north of the center, which is in better agreement with the HIRAD surface wind observations Fig. $6 \mathrm{~b}$ that have the maximum winds to the north-northwest of the center.
By contrast, the CNTL-NAVGEM forecast has the maximum winds at a larger radius to the east of the center.

Although this is a single case, the SCDI-NAVGEM initial conditions contributed to an improved NAVGEM track forecast to $48 \mathrm{~h}$. Given that global models typically underforecast the intensity of hurricanes because of their coarse resolution, the SCDI-NAVGEM analysis provided an excellent intensity forecast to $36 \mathrm{~h}$ in terms of both the large magnitude and the constant trend.

The upscaling of the SCDI analysis to NAVGEM also resulted in a better 24-h forecast of the inner-core surface winds and the size of the vortex (here the $18 \mathrm{~m} \mathrm{~s}^{-1}$ winds), which should lead to a better ocean surface wave forecast (not shown). This combination of a more accurate track, intensity, and vortex surface wind distribution is important for warnings for naval ships that must stay outside the $35-\mathrm{kt}$ wind radius and avoid $12-\mathrm{ft}$ seas associated with a hurricane. 


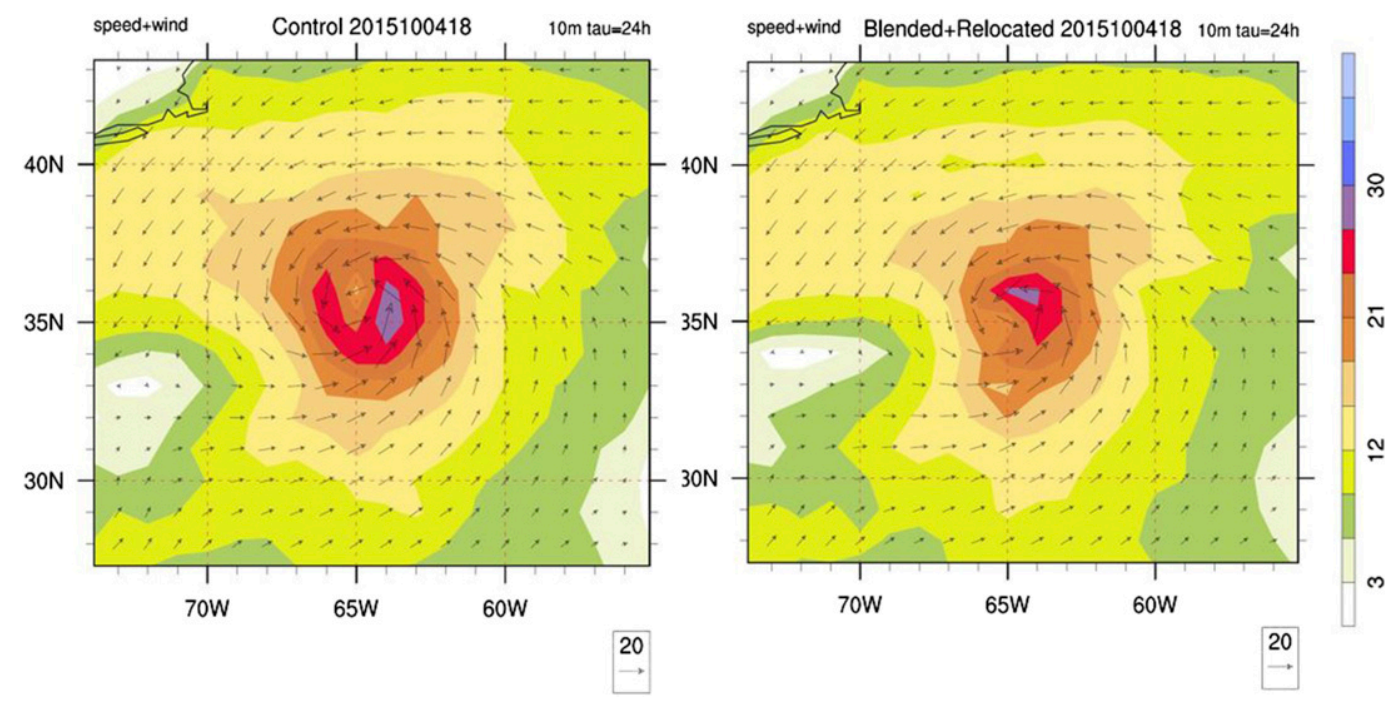

FIG. 15. Comparison of 24-h forecasts from 1800 UTC 4 Oct of the $10-\mathrm{m}$ wind speeds ( $\mathrm{m} \mathrm{s}^{-1}$; color scale on right) for the (a) CNTL-NAVGEM and (b) SCDI-NAVGEM initial conditions.

\section{Discussion}

The specific objective of this study has been to demonstrate the potential impacts on the U.S. Navy regional and global model analyses and forecasts with a novel technique for fully utilizing the nearly continuous (10- or 15-min interval) AMVs that are now possible from the new generation of geostationary meteorological satellites such as the Japanese Himawari-8 and the U.S. GOES-16. This first demonstration is carried out in the Atlantic region to take advantage of special AMV datasets from the TCI-15 field experiment along with HDSS dropwindsondes and HIRAD surface wind speeds for validation. Such a validation would not be possible in the western North Pacific because of the lack of such high-resolution dropwindsondes and surface wind speeds.

This demonstration is similar to the Velden et al. (2017) study in that testing is done at 6-h intervals. Whereas Velden et al. utilized hourly AMVs at $\pm 3 \mathrm{~h}$ of the target analysis times, the SCDI has been designed to utilize AMVs at 15-min intervals during the $6 \mathrm{~h}$ leading up to the target time. Due to restrictions employed in the HWRF initialization procedure, Velden et al. did not utilize any AMVs between 400 and $700 \mathrm{hPa}$ or over land. More importantly, the HWRF initialization eliminates analysis increments within $150 \mathrm{~km}$ of the center below $600 \mathrm{hPa}$ in order to maintain the enhanced vortex structure based on the National Hurricane Center analyzed position, intensity, and wind radii. By contrast, the SCDI technique calculates those TC characteristics via the dynamic and thermodynamic processes in the COAMPS-TC model in response to the AMVs that monitor the outflow and the environmental inflow every $15 \mathrm{~min}$. The role of the SAMURAI analysis is to spread that AMV information horizontally and vertically as increments relative to the background COAMPS-TC forecast wind field each $15 \mathrm{~min}$. Then the key to the success in this 3DVAR analysis is that in the COAMPS-TC dynamic initialization 90 time steps on the domain 3 grid and 30 time steps on the domain 2 grid are available to balance the mass field to those AMV-based SAMURAI wind increments during each 15-min interval. In this sense, the near-continuous utilization of the AMV information during the 6-h period resembles that of a 4DVAR approach. While it is likely the 15-min AMVs are appropriate for the inner domains of COAMPS-TC, it needs to be tested whether 30-min or even hourly AMVs would be adequate in domain 1 .

Even though the SCDI analyses began with a cold start in which the TC structure was represented by a bogus vortex, the surface wind speeds observed by HIRAD were reasonably replicated in the SCDI analysis after $6 \mathrm{~h}$. It was particularly encouraging that the vortex tilt of Joaquin at 1800 UTC 4 October inferred from the HDSS dropwinsondes was also well analyzed by SCDI. Whereas in a cold start of the COAMPS-TC the bogus vortex has no clouds, the SCDI generates a realistic TC-like cloud and relative humidity structure. Thus, a COAMPS-TC model integration from the 6-h SCDI analysis can begin smoothly without the initial intensity decay period that is inherent in a cold-start COAMPS-TC forecast as the moisture field and clouds of the TC are generated. 
For the COAMPS-TC version that is initialized with NAVGEM initial and lateral boundary conditions, a substantial improvement in the Joaquin track forecast is achieved with the SCDI analysis. Although the COAMPS-TC forecast from the SCDI analysis at 1800 UTC 4 October continued the rapid decay of Joaquin an additional $12 \mathrm{~h}$ rather than the abrupt transition to a constant $V_{\max }$ in the NHC best track, such a continued rapid decay seems more physically reasonable and there is some evidence from satellite imagery interpretation in support of that continued decay. Furthermore, the prediction of an extended period of near-constant MSLP during the period of constant $V_{\max }$ is physically reasonable compared to the continuous MSLP rises in the NHC best track.

The upscaling of the SCDI analysis valid at 1800 UTC 4 October into the NAVGEM initial conditions as an alternative to a synthetic TC vortex is successful in the sense that a more accurate track and inner-core winds, as well as a more realistic outer vortex structure, is achieved. Because the SCDI is a dynamic initialization, the NAVGEM intensity forecast will start more smoothly than will the ControlNAVGEM forecast with a static synthetic vortex. Further improvements of the intensity and intensity trend predictions are expected from multiple 6-h cycles during which the SCDI analyses are upscaled to the NAVGEM. Because these short-term NAVGEM forecasts will then be utilized as the initial and lateral boundary conditions for the COAMPS-TC model that is an integral part of the SCDI, this is an end-to-end approach for assimilating high-frequency AMVs that are now possible from the new-generation Himawari-8 and GOES-16 satellites.

Acknowledgments. This study would not have been possible without the tremendous effort on the part of the NASA WB-57 flight crew and mission support team in successfully carrying out difficult missions in Hurricane Joaquin under the excellent guidance provided by the TCI-15 mission planning team. Bob Creasey provided the vortex tilt calculations in Fig. 5, and Dan Cecil provided Fig. 6. Dave Stettner of CIMSS helped with the reprocessed AMV datasets. Eric Hendricks is supported by Office of Naval Research (ONR) Grant N0001417WX01042, Russell Elsberry and Melinda Peng by ONR Grant N00141712160, Chris Velden by ONR Grant N0001410116, Michael Bell and Eleanor Casas by ONR Grants N000141410118 and N000141613033, and Qingyun Zhao by ONRsponsored Research Project N0001415WX00849. Penny Jones provided excellent support in the manuscript preparation.

\section{REFERENCES}

Bell, M. M., M. T. Montgomery, and K. E. Emanuel, 2012: Air-sea enthalpy and momentum exchange at major hurricane wind speeds observed during CBLAST. J. Atmos. Sci., 69, 31973222, https://doi.org/10.1175/JAS-D-11-0276.1.

Berg, R., 2016: Hurricane Joaquin (AL112015) 28 September-7 October 2015. National Hurricane Center Tropical Cyclone Rep., 36 pp., www.nhc.noaa.gov/data/tcr/AL112015_Joaquin.pdf.

Cecil, D. J., and S. K. Biswas, 2017: Hurricane Imaging Radiometer (HIRAD) wind speed retrieval assessment with dropsondes. Tropical Cyclone Operations and Research Forum/71st Interdepartmental Hurricane Conf., Miami, FL, Office of the Federal Coordinator for Meteorology, http://www.ofcm.gov/meetings/TCORF/ihc17/Session_04/ 4-2-HIRAD_Cecil_TCORF2017web.pdf.

Creasey, R. L., and R. L. Elsberry, 2017: Tropical cyclone center positions from sequences of HDSS sondes deployed along high-altitude overpasses. Wea. Forecasting, 32, 317-325, https:// doi.org/10.1175/WAF-D-16-0096.1.

Doyle, J. D., and Coauthors, 2014: Tropical cyclone prediction using COAMPS-TC. Oceanography, 27, 104-115, https://doi.org/ 10.5670/oceanog.2014.72.

- , and Coauthors, 2017: A view of tropical cyclones from above: The Tropical Cyclone Intensity Experiment. Bull. Amer. Meteor. Soc., 98, 2113-2134, https://doi.org/10.1175/BAMS-D-16-0055.1.

Foerster, A. M., and M. M. Bell, 2017: Thermodynamic retrieval in rapidly rotating vortices from multiple-Doppler radar data. J. Atmos. Oceanic Technol., 34, 2353-2374, https://doi.org/ 10.1175/JTECH-D-17-0073.1.

Hendricks, E. A., M. S. Peng, X. Ge, and T. Li, 2011: Performance of dynamic initialization scheme in the Coupled OceanAtmosphere Mesoscale Prediction System for Tropical Cyclones (COAMPS-TC). Wea. Forecasting, 26, 650-663, https:// doi.org/10.1175/WAF-D-10-05051.1.

__, _ _ and T. Li, 2013: Evaluation of multiple dynamic initialization schemes for tropical cyclone prediction. Mon. Wea. Rev., 141, 4028-4048, https://doi.org/10.1175/ MWR-D-12-00329.1.

— R. L. Elsberry, C. S. Velden, A. C. Jorgensen, M. S. Jordan, and R. L. Creasey, 2018: Environmental factors and internal processes contributing to interrupted rapid decay of Hurricane Joaquin (2015). Wea. Forecasting, 33, 1251-1262, https:// doi.org/10.1175/WAF-D-17-0190.1.

Herrera, M. A., and Coauthors, 2018: Regionally enhanced global (REG) 4D-VAR: Methodology and global model performance. Mon. Wea. Rev., 146, 4015-4038, https://doi.org/ 10.1175/MWR-D-17-0228.1.

Hogan, T. F., and Coauthors, 2014: Navy global environmental model. Oceanography, 27, 116-125, https://doi.org/10.5670/ oceanog.2014.73.

Kim, M., H. M. Kim, J. W. Kim, S.-M. Kim, C. Velden, and B. Hoover, 2017: Effect of enhanced satellite-derived atmospheric motion vectors on numerical weather prediction in East Asia using an adjoint-based observation impact method. Wea. Forecasting, 32, 579-594, https://doi.org/ 10.1175/WAF-D-16-0061.1.

Ooyama, K. V., 2002: The cubic-spline transform method: Basic definitions and tests in a 1D single domain. Mon. Wea. Rev., 130, 2392 2415, https://doi.org/10.1175/1520-0493(2002)130<2392: TCSTMB $>2.0 . C O ; 2$.

Oyama, R., 2017: Relationship between tropical cyclone intensification and cloud-top outflow revealed by upper-tropospheric 
atmospheric motion vectors. J. Appl. Meteor. Climatol., 56, 28012819, https://doi.org/10.1175/JAMC-D-17-0058.1.

_, M. Sawada, and K. Shimoji, 2018: Diagnosis of tropical cyclone intensity and structure using upper tropospheric atmosphere motion vectors. J. Meteor. Soc. Japan, 96B, 3-16, https://doi.org/10.2151/jmsj.2017-024.

Purser, R. J., W.-S. Wu, D. F. Parrish, and N. M. Roberts, 2003: Numerical aspects of the application of recursive filters to variational statistical analysis. Part I: Spatially homogeneous and isotropic Gaussian covariances. Mon. Wea. Rev., 131, 1524-1535, https://doi.org/10.1175//1520-0493(2003)131<1524: NAOTAO $>2.0 . \mathrm{CO} ; 2$.

Schmit, T. J., P. Griffith, M. M. Gunshor, J. M. Daniels, S. J. Goodman, and W. J. Lebair, 2017: A closer look at the ABI on the GOES-R series. Bull. Amer. Meteor. Soc., 98, 681-698, https://doi.org/10.1175/BAMS-D-15-00230.1.

Velden, C., and Coauthors, 2005: Recent innovations in deriving tropospheric winds from meteorological satellites. Bull. Amer. Meteor. Soc., 86, 205-223, https://doi.org/10.1175/BAMS-86-2-205.

, W. E. Lewis, W. Bresky, D. Stettner, J. Daniels, and S. Wanzong, 2017: Assimilation of high-resolution satellitederived atmospheric motion vectors: Impact on HWRF forecasts of tropical cyclone track and intensity. Mon. Wea. Rev., 145, 1107-1125, https://doi.org/10.1175/MWR-D-16-0229.1.

$\mathrm{Xu}$, L., T. Rosmond, and R. Daley, 2005: Development of NAVDAS-AR: Formulation and initial tests of the linear problem. Tellus, 57A, 546-559, https://doi.org/10.3402/ tellusa.v57i4.14710. 